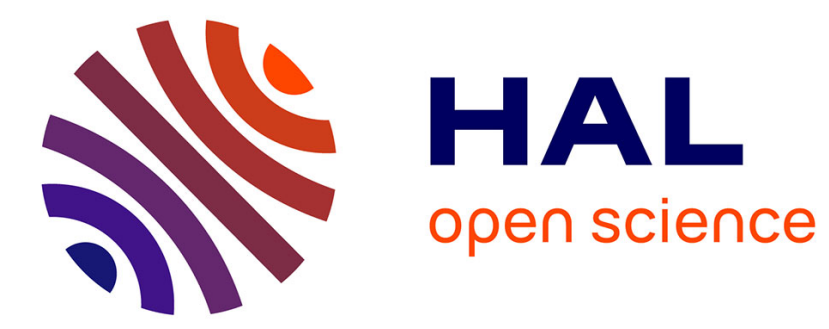

\title{
Almost split real forms for hyperbolic Kac-Moody Lie algebras
}

\author{
Hechmi Ben Messaoud
}

\section{To cite this version:}

Hechmi Ben Messaoud. Almost split real forms for hyperbolic Kac-Moody Lie algebras. Journal of Physics A: Mathematical and Theoretical, 2006, 39, pp.13659-13690. 10.1088/0305-4470/39/44/004 . hal-00020268

\section{HAL Id: hal-00020268 https://hal.science/hal-00020268}

Submitted on 8 Mar 2006

HAL is a multi-disciplinary open access archive for the deposit and dissemination of scientific research documents, whether they are published or not. The documents may come from teaching and research institutions in France or abroad, or from public or private research centers.
L'archive ouverte pluridisciplinaire HAL, est destinée au dépôt et à la diffusion de documents scientifiques de niveau recherche, publiés ou non, émanant des établissements d'enseignement et de recherche français ou étrangers, des laboratoires publics ou privés. 


\title{
ALMOST SPLIT REAL FORMS FOR HYPERBOLIC KAC-MOODY LIE ALGEBRAS
}

\author{
HECHMI BEN MESSAOUD
}

\begin{abstract}
A Borel-Tits theory was developped for almost split forms of symmetrizable Kac-Moody Lie algebras [J. of Algebra 171, 43-96 (1995)]. In this paper, we look to almost split real forms for symmetrizable hyperbolic KacMoody Lie algebras and we establish a complete list of these forms, in terms of their Satake-Tits index, for the strictly hyperbolic ones and for those which are obtained as (hyperbolic) canonical Lorentzian extensions of affine Lie algebras. These forms are of particular interest in theoretical physics because of their connection to supergravity theories.
\end{abstract}

Introduction. Since their appearance in the late 1960s, as generalizations of semi-simple complex Lie algebras, the (infinite-dimensional) Kac-Moody Lie algebras have played an increasingly crucial role in various areas of mathematics as well as theoretical physics. The hyperbolic Kac-Moody Lie algebras (which constitute a subclass of Lorentzian Kac-Moody algebras [22]) and some of their (almost split) real forms have appeared, besides the affine Kac-Moody algebras, in a variety of problems in the realms of string theory $([14],[13], \ldots)$ and supergravity theories $([34],[16], \ldots)$.

Almost split forms of symmetrizable Kac-Moody Lie algebras were studied in [28], [29], [30] and [2] for an arbitrary field of characteristic 0 : A Borel-Tits Theory was developed for these forms and a classification in the real case (in terms of the Satake-Tits index with the corresponding relative root system) was done for affine Lie algebras ([2]). In [27], G. Rousseau gave a realization, in terms of the loop algebras, for all the almost split real forms of affine Lie algebras. The same construction was done by $\mathrm{V}$. Back for an arbitrary field of characteristic 0 instead of the real field $([2], \S 5)$. Some forms (which may be almost anisotropic or almost compact in the real case) of symmetrizable Kac-Moody algebras are defined by generators and relations ([1], [10]). Almost compact real forms of affine Kac-Moody algebras were studied in [4] and [26] and entirely classified in [7]. The conjugate classes of their Cartan subalgebras were classified in [8].

This paper is devoted to the classification (in terms of the Satake-Tits index) of almost split real forms for some symmetrizable hyperbolic Kac-Moody Lie algebras (namely, the strictly hyperbolic Kac-Moody algebras and Kac-Moody Lie algebras which are obtained as (canonical) Lorentzian extensions of affine Lie algebras) which we consider the most met in supergravity theories ([16]).

The paper is organized as follows. In section 1, we recall the construction of (symmetrizable) Kac-Moody Lie algebras and groups from the so-called generalized Cartan matrices and we set the notations. We give also a description of the automorphisms group and the invariant bilinear form for any indecomposable and

2000 Mathematics Subject Classification. Primary 17B67; Secondary 83E50.

Key words and phrases. Hyperbolic Kac-Moody algebras, Lorentzian extensions, real forms. 
symmetrizable Kac-Moody algebra when the defining generalized Cartan matrix is non-singular (since in this case description is less complicated, and it is in particular valid for the hyperbolic case).

Sections 2 and 3 are mostly an exposition of known results on almost split forms, of symmetrizable Kac-Moody Lie algebras, and their relative root systems ([2], [3]) but rewritten here, in the real case, for hyperbolic Kac-Moody algebras. The main results are Theorem 2.2.7, Theorem 2.3.1 and Theorem 2.6.3 which are also valid for a symmetrizable Kac-Moody algebras with non-singular generalized Cartan matrix.

In section 4, we apply the results of sections 2 and 3 to give a complete list of almost split real forms for the subclass of strictly hyperbolic Kac-Moody Lie algebras and hyperbolic Kac-Moody Lie algebras which are obtained as (canonical) Lorentzian extensions of affine Lie algebras. Note that the classification of almost split real forms could be done for any symmetrizable hyperbolic Kac-Moody Lie algebra, but as the list of these algebras is long (at least 136), we have restricted ourselves to the selected subclass of hyperbolic algebras cited above.

\section{Preliminaries}

We recall the most important known facts about Kac-Moody algebras and groups, we add some facts in the hyperbolic case and set the notations.

1.1. Generalized Cartan matrices. An $n \times n$ matrix $A=\left(a_{i, j}\right)$ is called a generalized Cartan matrix if it satisfies :

(1) $a_{i, i}=2 \quad(i=1,2, \ldots, n)$

(2) $a_{i, j} \in\{0,-1,-2, \ldots\} \quad(i \neq j)$

(3) $a_{i, j}=0$ implies $a_{j, i}=0$.

The matrix $A$ is called decomposable if for a suitable permutation $\pi$ of $\{1,2, \ldots, n\}$ it takes the form $\left(a_{\pi(i), \pi(j)}\right)=\left(\begin{array}{cc}B & 0 \\ 0 & C\end{array}\right)$ where $B$ and $C$ are square matrices. If $A$ is not decomposable, it is called indecomposable.

The matrix $A$ is called symmetrizable if there exists an invertible diagonal matrix $D=\operatorname{diag}\left(d_{1}, d_{2}, \ldots, d_{n}\right)$ such that $D A$ is symmetric. The entries $d_{1}, d_{2}, \ldots, d_{n}$ can be choosen to be positive rational and, if moreover the matrix $A$ is indecomposable, they are unique up to a constant factor.

It was stated in [17] that the collection of indecomposable generalized Cartan matrices is divided into three mutually exclusive types : finite, affine and indefinite. A classification of these matrices in terms of their Dynkin diagrams is done for the finite and the affine type ones and it can be found (for example) in [17], chap 4. An indecomposable generalized Cartan matrix $A$ is called strictly hyperbolic (resp. hyperbolic) if it is of indefinite type and all of its principal submatrices have all their indecomposable constituents of finite (resp. finite or affine) type. This means that the Dynkin diagram corresponding to $A$ reverts to that of finite (resp. finite or affine) type upon deletion of one of the vertices.

The matrices $H_{s, t}=\left(\begin{array}{cc}2 & -s \\ -t & 2\end{array}\right)$ where $s$ and $t$ are positive integers such that $s t>4$, cover all the rank -2 generalized Cartan matrices of indefinite type, they are symmetrizable and strictly hyperbolic and they have been treated by Lepowsky and Moody in [20]. The Dynkin diagram corresponding to the strictly hyperbolic matrix $H_{s, t}$ (we may assume $0<s \leq t$ ) is the following : 


$$
H_{(s, t)}: \quad \stackrel{s}{:} \quad \stackrel{t}{2}
$$

The classification by Saçlioğlu ([31]) of hyperbolic Dynkin diagrams shows that there is no strictly hyperbolic (resp. hyperbolic) generalized Cartan matrix of order larger than 4 (resp. 10). The number of hyperbolic generalized Cartan matrices of order from 3 to 10 is necessarily finite (cf. [31]).

Note that a symmetrizable hyperbolic generalized Cartan matrix is non-singular and the corresponding symmetric matrix has signature $(++\ldots+-)$ cf. [23]. In particular, hyperbolic generalized Cartan matrices are Lorentzian.

1.2. Kac-Moody algebras and groups. (See [17] and [25]). Let $A=\left(a_{i, j}\right)$ be an $n \times n$ indecomposable and symmetrizable generalized Cartan matrix with a re-

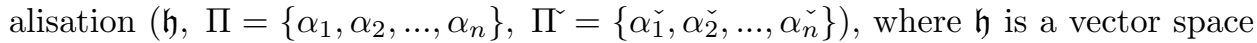
over the complex field $\mathbb{C}$ such that $\operatorname{dimh}=n+\operatorname{corank}(A), \Pi$ and $\Pi^{\sim}$ are linearly independent in $\mathfrak{h}^{*}$ and $\mathfrak{h}$ respectively such that $\left\langle\alpha_{j}, \alpha_{i}^{\check{i}}\right\rangle=a_{i, j}$. It follows that if $A$ is non-singular then $\Pi^{\vee}$ (resp. $\Pi$ ) is a basis of $\mathfrak{h}\left(\right.$ resp. $\left.\mathfrak{h}^{*}\right)$ and we denote by $P=\left(p_{1}, p_{2}, \ldots, p_{n}\right)$ the dual basis of $\Pi$ in $\mathfrak{h}$.

Let $\mathfrak{g}=\mathfrak{g}(A)$ be the complex Kac-Moody algebra associated to $A$ : it is generated by $\left\{\mathfrak{h}, e_{i}, f_{i} ; i=1,2, \ldots, n\right\}$ with the following relations

$$
\begin{array}{lll}
{[\mathfrak{h}, \mathfrak{h}]=0,} & {\left[e_{i}, f_{j}\right]=\delta_{i, j} \alpha_{i}} & (i, j=1,2, \ldots, n) ; \\
{\left[h, e_{i}\right]=\left\langle\alpha_{i}, h\right\rangle e_{i},} & {\left[h, f_{i}\right]=-\left\langle\alpha_{i}, h\right\rangle f_{i}} & (h \in \mathfrak{h}) ; \\
\left(\operatorname{ad} e_{i}\right)^{1-a_{i, j}}\left(e_{j}\right)=0, & \left(\operatorname{ad} f_{i}\right)^{1-a_{i, j}}\left(f_{j}\right)=0 & (i \neq j) .
\end{array}
$$

The Kac-Moody algebra $\mathfrak{g}=\mathfrak{g}(A)$ is called of finite, affine or indefinite type if the corresponding generalized Cartan matrix $A$ is.

The derived algebra $\mathfrak{g}^{\prime}$ of $\mathfrak{g}$ is generated by the Chevalley generators $e_{i}, f_{i}$; $(i=1,2, \ldots, n)$ and the center $\mathfrak{c}$ of $\mathfrak{g}$ lies in $\mathfrak{h}^{\prime}=\mathfrak{h} \cap \mathfrak{g}^{\prime}=\sum_{i=1}^{n} \mathbb{C} \alpha_{i}$. If the generalized Catan matrix $A$ is non-singular (that is the case when $A$ is of finite type or hyperbolic) then $\mathfrak{g}=\mathfrak{g}^{\prime}$ is a (finite or infinite)-dimensional simple Lie algebra and the center $\mathfrak{c}$ is trivial.

The Chevalley Cartan involution $\omega$ of $\mathfrak{g}$ is the involutive automorphism such that $\omega(h)=-h(h \in \mathfrak{h})$ and $\omega\left(e_{i}\right)=-f_{i}(i=1,2, \ldots, n)$

The subalgebra $\mathfrak{h}$ is a maximal ad $(\mathfrak{g})$-diagonalizable subalgebra of $\mathfrak{g}$, it is called the standard Cartan subalgebra of $\mathfrak{g}$. Let $\Delta=\Delta(\mathfrak{g}, \mathfrak{h})$ be the corresponding root system; then $\Pi$ is a root basis of $\Delta$ and $\Delta=\Delta^{+} \cup \Delta^{-}$, where $\Delta^{ \pm}=\Delta \cap \mathbb{Z}^{ \pm} \Pi$ is the set of positive (or negative) roots relative to the basis $\Pi$.

The Weyl group $W$ of $(\mathfrak{g}, \mathfrak{h})$ is generated by the fundamental reflections $r_{i}(i=$ $1,2, \ldots, n)$ defined by $r_{i}(h)=h-\left\langle\alpha_{i}, h\right\rangle \alpha_{i}$ for $h \in \mathfrak{h}$, it is a Coxeter group on $\left\{r_{1}, r_{2}, \ldots, r_{n}\right\}$ with length function $w \mapsto l(w), w \in W$. The Weyl group $W$ acts on $\mathfrak{h}^{*}$ and $\Delta$, we set $\Delta^{r e}=W(\Pi)$ (the real roots) and $\Delta^{i m}=\Delta \backslash \Delta^{r e}$ (the imaginary roots). Any root basis of $\Delta$ is $W$-conjugate to $\Pi$ or $-\Pi$. The opposite root basis $-\Pi$ is $W$-conjugate to $\Pi$ if and only if the generalized Cartan matrix $A$ is of finite type.

For $\alpha \in \Delta$, let $\mathfrak{g}_{\alpha}$ be the root space of $\mathfrak{g}$ corresponding to the root $\alpha$. We have the root space decomposition $\mathfrak{g}=\mathfrak{h} \oplus\left(\oplus_{\alpha \in \Delta} \mathfrak{g}_{\alpha}\right)$.

A Borel subalgebra of $\mathfrak{g}$ is a maximal completely solvable subalgebra. A parabolic subalgebra of $\mathfrak{g}$ is a (proper) subalgebra containing a Borel subalgebra. The standard positive (or negative) Borel subalgebra is $\mathfrak{b}^{ \pm}:=\mathfrak{h} \oplus\left(\oplus_{\alpha \in \Delta^{ \pm}} \mathfrak{g}_{\alpha}\right)$. A parabolic 
subalgebra $\mathfrak{p}^{+}\left(\operatorname{resp} . \mathfrak{p}^{-}\right)$containing $\mathfrak{b}^{+}\left(\operatorname{resp} . \mathfrak{b}^{-}\right)$is called positive (resp. negative) standard parabolic subalgebra of $\mathfrak{g}$; then there exists a subset $J$ of $\Pi$ (different from I) such that $\mathfrak{p}^{ \pm}=\mathfrak{p}^{ \pm}(J):=\left(\oplus_{\alpha \in \Delta_{J}} \mathfrak{g}_{\alpha}\right)+\mathfrak{b}^{ \pm}$, where $\Delta_{J}=\Delta \cap(\mathbb{Z} J)$, and we say that $\mathfrak{p}^{ \pm}$is of type $J$ (cf. [19]). If $J$ is of finite type (i.e $\Delta_{J}$ is finite) the standard parabolic subalgebra $\mathfrak{p}^{ \pm}$is said of finite type.

In [25], D.H. Peterson and V.G. Kac construct a group $G$, which is the connected and simply connected complex algebraic group associated to $\mathfrak{g}$ when $\mathfrak{g}$ is of finite type, depending only on the derived Lie algebra $\mathfrak{g}^{\prime}$ and acting on $\mathfrak{g}$ via the adjoint representation $\mathrm{Ad}: G \rightarrow \operatorname{Aut}(\mathfrak{g})$. It is generated by the one-parameter subgroups $U_{\alpha}=\exp \left(\mathfrak{g}_{\alpha}\right), \alpha \in \Delta^{r e}$, and $\left.\operatorname{Ad}\left(U_{\alpha}\right)=\exp \left(\operatorname{ad} \mathfrak{g}_{\alpha}\right)\right)$.

For each $i=1,2, \ldots, n$ there exists a monomorphism $\phi_{i}: S L_{2}(\mathbb{C}) \rightarrow G$ satisfying

$$
\phi_{i}\left(\left(\begin{array}{ll}
1 & t \\
0 & 1
\end{array}\right)\right)=\exp \left(t e_{i}\right), \quad \phi_{i}\left(\left(\begin{array}{ll}
1 & 0 \\
t & 1
\end{array}\right)\right)=\exp \left(t f_{i}\right), t \in \mathbb{C}
$$

Set $m_{i}=\phi_{i}\left(\left(\begin{array}{cc}0 & 1 \\ -1 & 0\end{array}\right)\right)=\exp \left(e_{i}\right) \exp \left(-f_{i}\right) \exp \left(e_{i}\right)=\exp \left(-f_{i}\right) \exp \left(e_{i}\right) \exp \left(-f_{i}\right)$; then $\operatorname{Ad}\left(m_{i}\right)$ induces the fundamental reflection $r_{i}$ on $\mathfrak{h}$ and we may always view an element of the Weyl group $W$ as an automorphism of $\mathfrak{g}$.

Let $N$ (resp. $H$ ) be the stabilizer (resp. fixator) of $\mathfrak{h}$ in $G$, then $H$ is a normal subgroup of $N$ and $N / H$ is isomorphic to the Weyl group $W$.

The center $Z(G)(=\operatorname{Ker}(\mathrm{Ad}))$ of $G$ is contained in $H$, it is isomorphic to $\operatorname{Hom}\left(\mathbb{Z}^{n} / A \mathbb{Z}^{n}, \mathbb{C}^{\times}\right)$and it is finite when the generalized Cartan matrix $A$ is nonsingular.

The Cartan subalgebras of $\mathfrak{g}$ are conjugated by $G$. If $\mathfrak{g}$ is not of finite type, there are exactly two conjugacy classes (under the adjoint action of $G$ ) of Borel subalgebras : $G \mathfrak{b}^{+}$and $G \cdot \mathfrak{b}^{-}$. A Borel subalgebra $\mathfrak{b}$ of $\mathfrak{g}$ which is $G$-conjugate to $\mathfrak{b}^{+}$(resp. $\mathfrak{b}^{-}$) is called positive (resp. negative). It follows that any parabolic subalgebra $\mathfrak{p}$ of $\mathfrak{g}$ is $G$-conjugate to a standard positive (or negative) parabolic subalgebra and we say that $\mathfrak{p}$ is positive (or negative).

1.3. Automorphisms of Kac-Moody algebras. We give here a description of the group $\operatorname{Aut}(\mathfrak{g})$ of automorphisms of $\mathfrak{g}$ when the indecomposable and symmetrizable generalized Cartan matrix $A$ is non-singular and of indefinite type (that is the

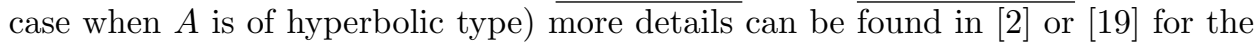
general case.

A linear or semi-linear automorphism $\sigma$ of $\mathfrak{g}$ over $\mathbb{C}$ is called of the first kind (resp. second kind) if it transforms a Borel subalgebra into a Borel subalgebra of the same (resp. opposite) sign. We denote $\operatorname{Aut}_{1}(\mathfrak{g})$ the subgroup of automorphisms of $\mathfrak{g}$ of the first kind. If $\sigma$ is of the first kind, then $\omega \sigma$ is of the second kind and one can see easily that $\operatorname{Aut}_{1}(\mathfrak{g})$ and $\omega \operatorname{Aut}_{1}(\mathfrak{g})$ form a partition of $\operatorname{Aut}(\mathfrak{g})$.

The adjoint group $\operatorname{Ad}(G)$ is a normal subgroup of $\operatorname{Aut}_{1}(\mathfrak{g})$ and $\operatorname{Aut}(\mathfrak{g})$. As the generalized Cartan matrix $A$ is assumed to be non-singular, the adjoint group is exactly the inner automorphisms group (denoted $\operatorname{Int}(\mathfrak{g})$ in [2]) in fact, the group $\tilde{H}=\operatorname{Hom}\left(\oplus_{i=1}^{n} \mathbb{Z} \alpha_{i}, \mathbb{C}^{\times}\right)$acts, in this case, on $\mathfrak{g}$ as $\operatorname{Ad}(H)$.

Let $\operatorname{Aut}(A)$ be the group of all permutations $\rho$ of $\{1,2, \ldots, n\}$ such that $a_{\rho(i), \rho(j)}=$ $a_{i, j}$. We view $\operatorname{Aut}(A)$ as a subgroup of the group $\operatorname{Aut}(\mathfrak{g})$ by requiring $\rho\left(e_{i}\right)=e_{\rho(i)}$ and $\rho\left(f_{i}\right)=f_{\rho(i)}$. Clearly, the group $\operatorname{Aut}(A)$ is contained in $\operatorname{Aut}_{1}(\mathfrak{g})$, it commutes to the Chevalley Cartan involution $\omega$ and we denote $\operatorname{Out}(A)$ the group $\{1, \omega\} \times \operatorname{Aut}(A)$. 
We have

$$
\begin{aligned}
& \operatorname{Aut}_{1}(\mathfrak{g})=\operatorname{Aut}(A) \ltimes \operatorname{Ad}(G) \\
& \operatorname{Aut}(\mathfrak{g})=\operatorname{Out}(A) \ltimes \operatorname{Ad}(G)
\end{aligned}
$$

1.4. The invariant bilinear form. (See [17]) We recall that the generalized Car$\tan$ matrix $A$ is supposed indecomposable and symmetrizable. There exists a nondegenerate $\operatorname{ad}(\mathfrak{g})$ - invariant symmetric $\mathbb{C}$-bilinear form $(.$, . $)$ on $\mathfrak{g}$, which is entirely determined by its restriction to $\mathfrak{h}$, such that

$$
\left(\widetilde{\alpha_{i}}, h\right)=\frac{\left(\widetilde{\alpha_{i}}, \alpha_{i}^{\check{i}}\right)}{2}\left\langle\alpha_{i}, h\right\rangle, \quad i=1,2, \ldots, n, h \in \mathfrak{h} .
$$

Set $d_{i}=\frac{2}{\left(\alpha_{\tilde{i}}, \alpha_{i}\right)}$ and $D=\operatorname{diag}\left(d_{1}, d_{2}, \ldots, d_{n}\right)$, then the matrix $D A$ is symmetric and we may thus assume that

$$
\left(\alpha_{\tilde{i}}^{\check{\alpha}}, \check{\alpha_{i}}\right) \text { is a positive rational for all } i \text {. }
$$

The form $(.,$.$) is clearly \operatorname{Ad}(G)$-invariant and invariant by the Chevalley Cartan involution $\omega$ ( since $\omega$ acts by -1 on $\mathfrak{h})$.

If moreover the generalized Cartan matrix $A$ is non-singular, then the invariant bilinear form $(.,$.$) satisfying the condition 1.4 .1$ is unique up to a positive rational factor. It follows that the form $(.,$.$) is \operatorname{Aut}(A)$-invariant; indeed, if $\rho$ is a diagram automorphism, then the invariant bilinear form $(\rho(x), \rho(y))$ satisfies the condition 1.4.1; hence, there exists a positive rational $\lambda$ such that $(\rho(x), \rho(y))=\lambda(x, y)$, $\forall x, y \in \mathfrak{g}$; but $\rho$ is of finite order and we have necessarily $\lambda=1$. Then we deduce from 1.3.1 that the bilinear form $(.,$.$) is \operatorname{Aut}(\mathfrak{g})$-invariant.

\section{Almost split REAl Forms}

From now on we suppose that the generalized Cartan matrix $A$ is indecomposable, symmetrizable and non-singular of indefinite type. The associated Kac-Moody Lie algebra $\mathfrak{g}$ is defined as in 1.2 .

2.1. Definitions and notations. A real form $\mathfrak{g}_{\mathbb{R}}$ of $\mathfrak{g}$ corresponds to a semiinvolution (or a conjugate-linear involution) $\sigma^{\prime}$ of $\mathfrak{g}$ such that $\mathfrak{g}_{\mathbb{R}}$ is the fixed point real subalgebra $\mathfrak{g}^{\sigma^{\prime}}$. The real form $\mathfrak{g}_{\mathbb{R}}$ is said almost split (resp. almost compact) if the corresponding semi-involution $\sigma^{\prime}$ is of the first kind (resp. second kind).

The real subalgebra of $\mathfrak{g}$ (viewed as a real Lie algebra) generated by $e_{i}, f_{i}, i=$ $1,2, \ldots, n$ is an almost split real form of $\mathfrak{g}$; it is called the standard split form and the corresponding semi-involution of the first kind $\sigma_{n}^{\prime}$ is called the standard normal semi-involution of $\mathfrak{g}$.

The standard Cartan semi-involution of $\mathfrak{g}$ is $\omega^{\prime}=\sigma_{n}^{\prime} \omega=\omega \sigma_{n}^{\prime}$, it is of the second kind and the corresponding almost compact form is the standard compact form of $\mathfrak{g}$. A Cartan semi-involution of $\mathfrak{g}$ is a $G$-conjugate of $\omega^{\prime}$.

Let $\mathfrak{g}_{\mathbb{R}}$ be a real form of $\mathfrak{g}$ and $\sigma^{\prime}$ the corresponding semi-involution of $\mathfrak{g}$. If $V$ is a $\sigma^{\prime}$-stable $\mathbb{C}$-subspace of $\mathfrak{g}$ we denote $V_{\mathbb{R}}:=V^{\sigma^{\prime}}$ the fixed point set of $\sigma^{\prime}$ in $V$. Conversely, if $V_{\mathbb{R}}$ is a $\mathbb{R}$-subspace of $\mathfrak{g}_{\mathbb{R}}$ we denote $V$ the $\sigma^{\prime}$-stable $\mathbb{C}$ - subspace $V_{\mathbb{R}} \otimes_{\mathbb{R}} \mathbb{C}$ of $\mathfrak{g}$. The semi-involution $\sigma^{\prime}$ acts on the Kac-Moody group $G$ associated to $\mathfrak{g}$ and we denote by $G_{\mathbb{R}}:=G^{\sigma^{\prime}}$ the fixed point subgroup of $\sigma^{\prime}$. 
A Cartan subalgebra of $\mathfrak{g}_{\mathbb{R}}$ is a subalgebra whose complexification is a Cartan subalgebra of $\mathfrak{g}$. A split toral subalgebra of $\mathfrak{g}_{\mathbb{R}}$ is an $\operatorname{ad}\left(\mathfrak{g}_{\mathbb{R}}\right)$-diagonalizable subalgebra. Any split toral subalgebra $\mathfrak{t}_{\mathbb{R}}$ of $\mathfrak{g}_{\mathbb{R}}$ is contained in a Cartan subalgebra $\mathfrak{h}_{\mathbb{R}}$. If $\mathfrak{t}_{\mathbb{R}}$ is a maximal split toral subalgebra, then $\mathfrak{h}_{\mathbb{R}}$ is said a maximally split (or maximally noncompact) Cartan subalgebra.

2.2. The index of an almost split real form. A Borel-Tits Theory of almost split $\mathbb{K}$-forms of Kac-Moody Lie algebras, where $\mathbb{K}$ is an arbitrary field of characteristic 0, was developed by G. Rousseau in [28], [29] and [2]. We recall (and rewrite in the real case) the main results on these forms for the class of Kac-Moody algebras that we have fixed above.

Proposition 2.2.1. Let $\mathfrak{g}_{\mathbb{R}}$ be a real form of $\mathfrak{g}$ and $\sigma^{\prime}$ the corresponding semiinvolution. Then $\mathfrak{g}_{\mathbb{R}}$ is almost split iff $\sigma^{\prime}$ stabilizes a proper parabolic subalgebra of $\mathfrak{g}$. In particular, almost compact real forms of $\mathfrak{g}$ are almost $\mathbb{R}$-anisotropic.

If the real form $\mathfrak{g}_{\mathbb{R}}$ is almost split then $\sigma^{\prime}$ stabilizes a finite type parabolic subalgebra $\mathfrak{p}$ of $\mathfrak{g}$. If $\mathfrak{p}$ is a Borel subalgebra, then the real form $\mathfrak{g}_{\mathbb{R}}$ is split or quasi-split.

Theorem 2.2.2. Let $\mathfrak{g}_{\mathbb{R}}$ be an almost split real form of $\mathfrak{g}$ and $\epsilon=+$ or - , then the group $G_{\mathbb{R}}$ is transitive on pairs $\left(\mathfrak{t}_{\mathbb{R}}, \mathfrak{p}_{\mathbb{R}}^{\epsilon}\right)$ where $\mathfrak{t}_{\mathbb{R}}$ is a maximal split toral subalgebra of $\mathfrak{g}_{\mathbb{R}}$ and $\mathfrak{p}_{\mathbb{R}}^{\epsilon}$ is a minimal parabolic subalgebra of $\mathfrak{g}_{\mathbb{R}}$ of sign $\epsilon$ and containing $\mathfrak{t}_{\mathbb{R}}$.

Definition 2.2.3. The rank of $\mathfrak{g}_{\mathbb{R}}$ is the common $\mathbb{R}$-dimension $r$ of its maximal split toral subalgebras.

Remark 2.2.4. The centralizer $\mathfrak{z}_{\mathbb{R}}$ of $\mathfrak{t}_{\mathbb{R}}$ in $\mathfrak{g}_{\mathbb{R}}$ is the Levi subalgebra of $\mathfrak{p}_{\mathbb{R}}^{\epsilon}$, its derived algebra $\mathfrak{l}_{\mathbb{R}}$ is a compact real semi-simple Lie algebra.

The maximal split toral subalgebra $\mathfrak{t}_{\mathbb{R}}$ is contained in a unique [up to a conjugation by $Z_{G_{\mathbb{R}}}\left(\mathfrak{t}_{\mathbb{R}}\right)$ ] maximally split Cartan subalgebra $\mathfrak{h}_{\mathbb{R}}$ of $\mathfrak{g}_{\mathbb{R}}$. In particular the maximally split Cartan subalgebras of $\mathfrak{g}_{\mathbb{R}}$ are $G_{\mathbb{R}}-$ conjugate.

Take a $G$-conjugate of $\sigma^{\prime}$, we may assume that $\mathfrak{h}\left(=\mathfrak{h}_{\mathbb{R}} \otimes \mathbb{C}\right)$ is the standard Cartan subalgebra of $\mathfrak{g}$ and $\mathfrak{p}^{\epsilon}=\mathfrak{p}^{\epsilon}(X)$ is a finite type standard parabolic subalgebra of $\operatorname{sign} \epsilon$. Therefore, the two opposite parabolic subalgebras $\mathfrak{p}^{+}(X)$ and $\mathfrak{p}^{-}(X)$ are $\sigma^{\prime}$-stable and we have

$$
\mathfrak{t} \subset \mathfrak{h} \subset \mathfrak{b}^{+} \subset \mathfrak{p}^{+}(X)
$$

Thus the pair $\left(\mathfrak{h}, \mathfrak{b}^{+}\right)$[resp. $\left.\left(\mathfrak{t}_{\mathbb{R}}, \mathfrak{p}_{\mathbb{R}}^{+}(X)\right)\right]$ is called a standardization of $\mathfrak{g}$ [resp. $\mathfrak{g}_{\mathbb{R}}$ ] and we say that the standardization $\left(\mathfrak{h}, \mathfrak{b}^{+}\right)$is compatible with $\left(\mathfrak{t}_{\mathbb{R}}, \mathfrak{p}_{\mathbb{R}}^{+}(X)\right)$.

Definition 2.2.5. [The $*$-action of $\sigma^{\prime}$ ] Actually, the derived Levi subalgebra $\mathfrak{l}$ is that of $\mathfrak{p}^{+}(X)$, it is the subalgebra $\mathfrak{g}_{X}$ generated by $\mathfrak{g}_{\alpha}, \alpha \in \Delta_{X}$. Note that $\sigma^{\prime}\left(\mathfrak{b}^{+}\right)$, as $\mathfrak{b}^{+}$, is a positive Borel subalgebra satisfying the condition 2.2.1; hence there exists $w_{X} \in W_{X}$ [the Weyl group of $\Delta(\mathfrak{h}, \mathfrak{l})$ viewed as a subgroup of $\left.W\right]$ such that $w_{X} \sigma^{\prime}\left(\mathfrak{b}^{+}\right)=\mathfrak{b}^{+}$and thus $w_{X} \sigma^{\prime}$ induces on $\Pi$ an involutive (or a trivial) diagram automorphism $\sigma^{*}$ called the $*$-action of $\sigma^{\prime}$.

One knows ([2]; Prop. 2.7) that $\mathfrak{t}$ is the subspace of $\mathfrak{h}$ defined by the following equations :

$$
\alpha(h)=0 \forall \alpha \in X, \text { and } \alpha(h)=\beta(h) \text { if } \alpha, \beta \in \Pi \text { satisfy } \beta=\sigma^{\prime *}(\alpha)
$$

The restriction of $\sigma^{\prime}$ to the derived Levi subalgebra $\mathfrak{l}$ is compact and we may assume that $\sigma^{\prime}$ coincides with the standard Cartan semi-involution $\omega^{\prime}$ on $\mathfrak{l}$. In particular $\sigma^{\prime}$ commutes with $w_{X}$ on $\mathfrak{h}$ and so $w_{X}^{2}=1_{W}$ is the unity of $W$. 
Definition 2.2.6. [The index of $\mathfrak{g}_{\mathbb{R}}$ ] The Satake-Tits index of $\mathfrak{g}_{\mathbb{R}}$ is the data consisting of the Dynkin diagram of $\mathfrak{g}$, the $*$-action of $\sigma^{\prime}$ on it and $X=\operatorname{type}\left(\mathfrak{p}^{+}(X)\right)$.

Schematically, the Satake-Tits index is the so-called Satake-Tits diagram on which the roots of $X$ are denoted by white circles $\circ$ and those of $\Pi \backslash X$ are denoted by black circles $\bullet$. If $\sigma^{\prime *}\left(\alpha_{i}\right)=\alpha_{j}$ then it will be indicated by $\stackrel{0}{i}_{i}^{j} \quad$ (or

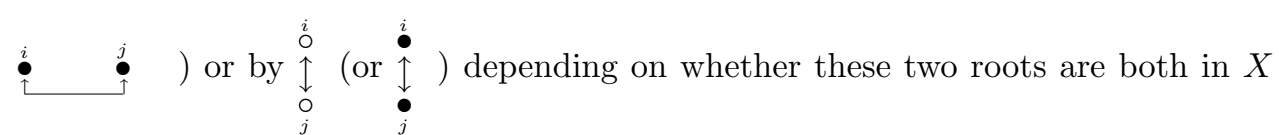
or in $\Pi \backslash X$ and on their positions on the Dynkin diagram.

Let us notice that the colouring of the vertices is chosen here so that the SatakeTits diagram for the split form is the Dynkin diagram of its complexified algebra (with black vertices); it differs from that of Helgason ([15], Chap X) where the roots of $X$ are black and those of $\Pi \backslash X$ are white.

As the derived Levi subalgebra $l$ has a unique compact form (up to a conjugation) we have as in the classical case ([35], [32]) :

Theorem 2.2.7. [2], Théorème 2.8] The knowledge of the Satake-Tits index determines the real form $\mathfrak{g}_{\mathbb{R}}$ up to an $\mathbb{R}$-isomorphism.

2.3. The classification problem and the admissible indices. In practice, the problem is to tell whether such an index comes from an almost split real form of $\mathfrak{g}$ or not. An index coming from a real form will be said admissible. To recognize admissible indices one established in [2] a one to one correspondence between conjugacy classes (under $\operatorname{Ad}(G)$ or $\operatorname{Aut}(\mathfrak{g})$ ) of almost split real forms and those of involutions of the second kind of $\mathfrak{g}$.

The semi-involution $\sigma^{\prime}$ and the standard Cartan semi-involution $\omega^{\prime}$ are assumed to stabilize the same Cartan subalgebra $\mathfrak{h}$ (which is maximally split for $\sigma^{\prime}$ ). Using an argument going back to Elie Cartan, one proved that there exists a unique (up to $H^{\sigma^{\prime}}$-conjugation) Cartan semi-involution which stabilizes $\mathfrak{h}$ and commutes to $\sigma^{\prime}$ (see [18] or [26]). By conjugating by $H$, one may assume that $\sigma^{\prime}$ commutes to $\omega^{\prime}$. Then $\sigma:=\sigma^{\prime} \omega^{\prime}$ is a $\mathbb{C}$-linear involution of the second kind of $\mathfrak{g}$. Hence $\mathfrak{t}=\mathfrak{h}^{-\sigma}$ is a maximal $\sigma$-split toral subalgebra of $\mathfrak{g}$ and the minimal $\sigma^{\prime}$-stable parabolic subalgebra $\mathfrak{p}^{+}(X)$ is $\sigma$-split, within the meaning of Kac and Wang [19], and it is minimal for this property. In particular the standardization $\left(\mathfrak{h}, \mathfrak{b}^{+}\right)$is a split pair for $\sigma$ and thus the restriction of $\sigma$ to $\mathfrak{l}\left(=\mathfrak{g}_{X}\right)$ is trivial, cf. ([19], §5) or $([2], \S 4)$. The main result of Kac and Wang on involutions of the second kind is that split pairs for $\sigma$ are $G^{\sigma}$-conjugate $([19], 5.32)$. Consequently, we have the following result:

Theorem 2.3.1. [[2], Théorème 4.4] consider :

(1) the semi-involutions of the first kind $\sigma^{\prime}$ of $\mathfrak{g}$,

(2) the involutions of the second kind $\sigma$ of $\mathfrak{g}$,

(3) the relation : $\sigma^{\prime} \sim \sigma$ iff:

(a) $\sigma^{\prime} \sigma=\sigma \sigma^{\prime}$ is a Cartan semi-involution,

(b) $\sigma^{\prime}$ and $\sigma$ stabilize a same Cartan subalgebra $\mathfrak{a}$,

(c) $\mathfrak{a}$ is contained in a minimal $\sigma^{\prime}$-stable parabolic subalgebra $\mathfrak{p}$ of $\mathfrak{g}$.

Then this relation induces a one to one correspondence between conjugacy classes under $\operatorname{Ad}(G)$ [resp. Aut $(\mathfrak{g})]$ of semi-involutions of the first kind and those of involutions of the second kind of $\mathfrak{g}$. 
Remark 2.3.2. The condition (c) in the theorem is equivalent to require that $\mathfrak{a}^{\sigma^{\prime}}$ is a maximally split Cartan subalgebra of $\mathfrak{g}^{\sigma^{\prime}}$.

The involution of the second kind $\sigma$ acts as the Cartan semi-involution $\sigma \sigma^{\prime}$ on the real form $\mathfrak{g}_{\mathbb{R}}$ corresponding to $\sigma^{\prime}$, it is called the Cartan involution of $\mathfrak{g}_{\mathbb{R}}$, and we have the corresponding Cartan decompostion :

$$
\mathfrak{g}_{\mathbb{R}}=\mathfrak{k} \oplus \mathfrak{p}
$$

In ([9], Proposition 2.3) we proved that the Cartan involutions of $\mathfrak{g}_{\mathbb{R}}$ (or equivalently, the Cartan semi-involutions of $\mathfrak{g}$ commuting to $\sigma^{\prime}$ ) are $G_{\mathbb{R}}-$ conjugate.

2.4. Involutions of the second kind. Let $\sigma$ be an involution of the second kind of $\mathfrak{g}$. We may assume that $\left(\mathfrak{h}, \mathfrak{b}^{+}\right)$is a split pair, so there exists $X \subset \Pi$ of finite type such that $\sigma\left(\Delta^{+}\right) \cap \Delta^{+}=\Delta_{X}^{+}$and thus $\mathfrak{p}^{+}(X)$ is a minimal $\sigma$-split parabolic subalgebra. Then $\sigma$ can be written in this way (see [19], 4.39) :

$$
\sigma=\tau \omega \operatorname{Ad}\left(n_{X}\right) \operatorname{Ad}(s)
$$

where

i) $\tau$ is an involutive diagram automorphism stabilizing $X$ and $\omega$ is the standard Cartan involution.

ii) $n_{X} \in N^{\tau} \cap N^{\omega} \cap G_{X}$ such that its image in $W_{X}$ is the longest element $w_{0}(X)$, where $G_{X}$ is the subgroup of $G$ generated by the $U_{\alpha}, \alpha \in \Delta_{X}$, and $W_{X}$ the subgroup of $W$ generated by the $r_{i}, i \in X$.

iii) $s \in H^{\sigma}$.

To determine $A d(s)$, one needs to know the restriction of $A d\left(n_{X}\right)$ on $\mathfrak{g}_{X}$ and the action of $A d\left(n_{X}\right)^{2}$ on $\mathfrak{g}$. From the condition $\sigma \mid \mathfrak{g}_{X}=1$, one gets that $w_{0}(X)$ acts on $\mathfrak{h}_{X}\left(:=\mathfrak{h} \cap \mathfrak{g}_{X}\right)$ as $(-\tau)$ [i.e $\left.\tau \mid \mathfrak{h}_{X}=-w_{0}(X)\right]$.

2.5. How to choose $n_{X}$ ? By using the results of Tits on the word problem ([36] or [2]) one gives in [2] an explicit formula of $n_{X}$ and then one shows that the restriction of $A d\left(n_{X}\right)$ on $\mathfrak{g}_{X}$ is equal to that of $\tau \omega$ (see [2], 4.9). More precisely, for any reduced expression $\left(r_{i_{1}} r_{i_{2}} \ldots r_{i_{k}}\right)$ of the longest element $w_{0}(X)$ of $W_{X}$, the element $n_{X}:=\left(m_{i_{1}} m_{i_{2}} \ldots m_{i_{k}}\right) \in N^{\tau} \cap N^{\omega} \cap G_{X}$ does'nt depend on the choice of the reduced expression of $w_{0}(X)$ and the automorphism $A d\left(n_{X}\right)$ coincides with the traditional prolongation of $w_{0}(X)$ in an automorphism of $\mathfrak{g}$ of order dividing 4 (see [2], 4.7 and 4.8). Thus it is shown that:

$$
A d\left(n_{X}\right)^{2}=w_{0}(X)^{2}=\exp \left(i \pi \operatorname{ad}\left(2 \rho_{\check{X}}\right)\right)
$$

where $2 \rho_{\check{X}}$ is the sum of the positive coroots of $\Delta_{X}$ (see [2], 4.10.3).

In table 1 below we give, for each complex simple Lie algebra, a reduced expression of the longest element $w_{0}$ of the corresponding Weyl group, by noting that in the case $A_{n}, n \geq 1$, we have :

$$
w_{0}(X)=\left(r_{1} r_{2} \ldots r_{n}\right) \cdot\left(r_{1} \ldots r_{n-1}\right) \ldots\left(r_{1} r_{2}\right) \cdot r_{1}
$$

and that in all the other cases the Coxeter number $h$ is even, and thus $w_{0}(X)=$ $(c)^{\frac{h}{2}}$, where $c$ is a selected Coxeter transformation such that $(c)^{\frac{h}{2}}$ transforms the fundamental chamber into its opposite (cf. [12], chap V $\S 6$, Proposition 2). In the same table we give also the expression with the $\alpha_{i}$ (extracted from the "Planches de Bourbaki " [12]) of the sum of the positive coroots $2 \check{\rho}$.

In our situation, we choose for $n_{X}$ the product of the elements corresponding to the connected components of $X$, and those are the products obtained from the table 1 if one replaces $r_{i}$ by $m_{i}=\exp \left(e_{i}\right) \exp \left(-f_{i}\right) \exp \left(e_{i}\right)$. 
Table 1

\begin{tabular}{|c|c|c|c|}
\hline Name & Dynkin diagram & $w_{0}$ & $2 \rho^{\check{2}}$ \\
\hline$A_{n}, n \geq 1$ & $\stackrel{1}{\bullet} \bullet \bullet-\ldots \cdots-\bullet^{n}$ & $\begin{array}{l}\left(r_{1} r_{2} \ldots r_{n}\right)\left(r_{1} r_{2} \ldots r_{n-1}\right. \\
) \ldots \ldots\left(r_{1} r_{2}\right) r_{1}\end{array}$ & $\begin{array}{l}n \alpha_{1}+2(n-1) \alpha_{2}+\ldots+i(n-i+1) \alpha_{\check{i}}+\ldots \\
\ldots+n \alpha_{\check{n}}\end{array}$ \\
\hline$B_{n}, n \geq 3$ & ${ }^{1}-{ }^{2}-\ldots \ldots-\bullet->^{n}$ & $\left(r_{1} r_{2} \ldots r_{n}\right)^{n}$ & $\begin{array}{l}2 n \alpha_{\check{1}}+\ldots+i(2 n-i+1) \alpha_{\check{i}}+\ldots \\
\ldots+(n-1)(n+2) \alpha_{n \check{-1}}+\frac{n(n+1)}{2} \alpha_{n}\end{array}$ \\
\hline$C_{n}, n \geq 2$ & $\stackrel{1}{1}-2$ & $\left(r_{1} r_{2} \ldots r_{n}\right)^{n}$ & $\begin{array}{l}(2 n-1) \alpha_{\check{1}}+\ldots+i(2 n-i) \alpha_{\check{i}}+\ldots \\
\ldots+(n-1)(n+1) \alpha_{n \check{-}}+n^{2} \alpha_{\check{n}} \\
\end{array}$ \\
\hline$D_{n}, n \geq 4$ & $\bullet^{n-1}$ & $\left(r_{1} r_{2} \ldots r_{n}\right)^{n-1}$ & $\begin{array}{l}2(n-1) \alpha_{\check{1}}+\ldots+i(2 n-i-1) \alpha_{i}+\ldots \\
\quad \ldots+\frac{n(n-1)}{2}\left(\alpha_{n} \check{-1}+\alpha_{\check{n}}\right)\end{array}$ \\
\hline$E_{6}$ & $?_{2}^{1}$ & $\left(r_{1} r_{2} r_{3} r_{4} r_{5} r_{6}\right)^{6}$ & $\begin{array}{l}2\left(8 \alpha_{1}+11 \alpha_{2} \check{2}+15 \alpha_{\check{3}}+21 \alpha_{\breve{4}}+\right. \\
\left.15 \alpha_{5}+8 \alpha_{6}\right)\end{array}$ \\
\hline$E_{7}$ & 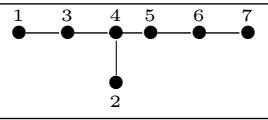 & $\left(r_{1} r_{2} r_{3} r_{4} r_{5} r_{6} r_{7}\right)^{9}$ & $\begin{array}{l}34 \alpha_{\check{1}}+49 \alpha_{\check{2}}+66 \alpha_{\check{3}}+96 \alpha_{4}+ \\
75 \alpha_{\check{5}}+52 \alpha_{\check{6}}+27 \alpha_{\check{7}}\end{array}$ \\
\hline$E_{8}$ & $\int_{2}^{1}$ & $\left(r_{1} r_{2} r_{3} r_{4} r_{5} r_{6} r_{7} r_{8}\right)^{15}$ & $\begin{array}{c}2\left(46 \alpha_{1}+68 \alpha_{2}+91 \alpha_{3}+135 \alpha_{\check{4}}+\right. \\
\left.110 \alpha_{5}+84 \alpha_{\check{6}}+57 \alpha_{\overline{7}}+29 \alpha_{\check{8}}\right)\end{array}$ \\
\hline$F_{4}$ & 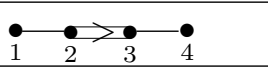 & $\left(r_{1} r_{2} r_{3} r_{4}\right)^{6}$ & $22 \alpha_{1}+42 \alpha_{2}+30 \alpha_{3}+16 \alpha_{4}$ \\
\hline$G_{2}$ & $\Longrightarrow 2$ & $\left(r_{1} r_{2}\right)^{3}$ & $2\left(5 \alpha_{1}+3 \alpha_{2}\right)$ \\
\hline
\end{tabular}

2.6. What is $\operatorname{Ad}(s)$ then ? Using the fact that $\sigma \mid \mathfrak{g}_{X}=1$, one can write (see the proof of 4.10 .4 in [2]) :

$$
\operatorname{Ad}(s)=\exp \left(i \pi \operatorname{ad}\left(\sum_{j \in \Omega} \epsilon_{j}\left(p_{j}-p_{\tau(j)}\right)\right)\right)
$$

where $\Omega$ is a complete set of representatives of the $\tau$-orbits in $\Pi \backslash X$ and $\epsilon_{j} \in \mathbb{Q}$ $(j \in \Omega)$ such that :

$$
2 \rho_{\check{X}}+\sum_{j \in \Omega} 2 \epsilon_{j}\left(p_{j}-p_{\tau(j)}\right) \quad \in 2 \mathbb{Z} P=\bigoplus_{i \in I} 2 \mathbb{Z} p_{i}
$$

here $I$ denotes the index set $\{1,2, \ldots, n\}$ and for any subset $X$ of $\Pi$ we confuse sometimes $X$ with the subset $I_{X}:=\left\{i \in I ; \alpha_{i} \in X\right\}$ of $I$.

Let $j \in \Omega$ and $u \in \mathbb{Z}$, then by conjugating $\sigma$ by $\left.\exp \left(\frac{\mathrm{i} \pi}{2} \operatorname{ad}\left(\mathrm{u}_{\left(\mathrm{p}_{\mathrm{j}}\right.}+\mathrm{p}_{\tau(\mathrm{j})}\right)\right)\right) \in \mathrm{Z}_{\mathrm{H}^{\omega^{\prime}}}\left(\mathfrak{g}_{\mathrm{X}}\right)$ one can replace $\epsilon_{j}$ by $\epsilon_{j}+u$ without thus modifying the others $\epsilon_{i}$, then $\forall j \in \Omega$, the element $\epsilon_{j} \in \frac{1}{2} \mathbb{Z}$ may be modified modulo $\mathbb{Z}$.

From 2.6.1 one sees that the class of $2 \rho_{\check{X}}$ in $\mathbb{Z} P / 2 \mathbb{Z} P$ has a representative in $\left(\mathbb{Z} P_{\Pi \backslash X}\right)^{(-\tau)}$, where $P_{\Pi \backslash X}=\left\{p_{i} ; \alpha_{i} \notin X\right\}$. And by the preceding result, for any such representative $2 \bar{\rho}_{X}$, one may modify the $\epsilon_{j}$ in order that :

$$
2 \bar{\rho}_{X}=\sum_{j \in \Omega} 2 \epsilon_{j}\left(p_{j}-p_{\tau(j)}\right)
$$

Hence we have the following results : 
Proposition 2.6.1. Conjugating by $Z_{H^{\omega^{\prime}}}\left(\mathfrak{g}_{X}\right)$, we may assume $\operatorname{Ad}(s)=\exp \left(\frac{\mathrm{i} \pi}{2} \operatorname{ad}\left(2 \bar{\rho}_{\mathrm{X}}\right)\right)$ and this yields

$$
\sigma=\tau \omega \operatorname{Ad}\left(\mathrm{n}_{\mathrm{X}}\right) \exp \left(\frac{\mathrm{i} \pi}{2} \operatorname{ad}\left(2 \bar{\rho}_{\mathrm{X}}\right)\right)
$$

Remark 2.6.2. Any involution of the second kind $\sigma$ taking the form 2.6.2 and the associated semi-involution of the first kind $\sigma^{\prime}:=\sigma \omega^{\prime}=\tau \sigma_{n}^{\prime} \operatorname{Ad}\left(n_{X}\right) \exp \left(\frac{i \pi}{2} \operatorname{ad}\left(2 \bar{\rho}_{X}\right)\right)$ are called standard. Any involution (resp. semi-involution) of the second (resp. the first) kind is $G$-conjugate to a standard one. Some involutions of the second kind (which are not all standard) are considered in [24] by Pati, Parashar and Kaushal to give the corresponding Iwasawa decomposition for some hyperbolic Kac-Moody Lie algebras.

Let $\sigma^{\prime}$ be a standard semi-involution of the first kind and $\mathfrak{g}_{\mathbb{R}}$ the corresponding almost split real form. Then the involutive diagram automorphism $\tau$ induces the *-action of $\sigma^{\prime}$ and thus the Satake-Tits index of $\mathfrak{g}_{\mathbb{R}}$ is entirely determined by the data $(\tau, X)$. Summarising, we get the following result :

Theorem 2.6.3. Let $X \subset \Pi$ be of finite type and $\tau$ an involutive diagram automorphism stabilizing $X$. Then the couple $(\tau, X)$ corresponds to an admissible index iff $\tau \mid \mathfrak{h}_{X}=-w_{0}(X)$ and the class of $2 \rho_{\check{X}}$ modulo $2 \mathbb{Z} P$ has a $(-\tau)$-fixed representative in $\mathbb{Z} P_{\Pi \backslash X}$. In particular, if $\tau$ is trivial (on $\left.\Pi \backslash X\right)$, then $\operatorname{Ad}\left(n_{X}\right)$ should be an involution, i.e. $2 \rho_{\check{X}}$ has to be in $2 \mathbb{Z} P$.

\section{The RELATIVE ROOT SYSTEM}

Let $\sigma^{\prime}$ be a semi-involution of the first kind and $\mathfrak{g}_{\mathbb{R}}$ the corresponding almost split real form. We may assume that $\sigma^{\prime}$ is standard (see 2.6.2) and thus $\sigma=$ $\sigma^{\prime} \omega^{\prime}=\omega^{\prime} \sigma^{\prime}$ is the Cartan involution of $\mathfrak{g}_{\mathbb{R}}$. Hence $\mathfrak{h}_{\mathbb{R}}$ is a $\sigma-$ stable maximally split Cartan subalgebra of $\mathfrak{g}_{\mathbb{R}}$ and $\mathfrak{a}_{\mathbb{R}}:=\mathfrak{h}_{\mathbb{R}}^{-\sigma}$ is a $\sigma-$ stable maximal split toral subalgebra. The group $K:=G_{\mathbb{R}}^{\sigma}$ is transitive on the set of $\sigma-$ stable maximally split Cartan subalgebras (resp. $\sigma-$ stable maximal split toral subalgebras) of $\mathfrak{g}_{\mathbb{R}}$ (cf.[9], Proposition 2.6).

Warning : Note that $\mathfrak{a}_{\mathbb{R}}$ is a maximal abelian subspace of $\mathfrak{p}$ (i.e. a Cartan subspace) but, in contrast to the classical case of real semi-simple Lie algebras, an element of $\mathfrak{p}$ is not necessarily ad $-\mathfrak{g}_{\mathbb{R}}$ diagonalizable; moreover a maximal abelian subspace of $\mathfrak{p}$ may be infinite dimensional, hence maximal abelian subspaces of $\mathfrak{p}$ are not $K$-conjugate.

The (infinite) relative root system $\Delta^{\prime}:=\Delta^{\prime}\left(\mathfrak{g}_{\mathbb{R}}, \mathfrak{a}_{\mathbb{R}}\right)=\left\{\alpha^{\prime}=\alpha \mid \mathfrak{a}_{\mathbb{R}} \neq 0 ; \alpha \in \Delta(\mathfrak{g}, \mathfrak{h})\right\}$ is more surprising, it was studied by Nicole Bardy in ([2], §3) (see also [3], §6). In our situation, we have explicitly :

$$
\mathfrak{a}_{\mathbb{R}}=\bigoplus_{i \in \Omega} \mathbb{R}\left(p_{i}+p_{\tau i}\right)
$$

where $\Omega$ is, as in $\S 2.6$, a complete set of representatives of the $\tau$-orbits in $I \backslash I_{X}$. It follows that the rank of $\mathfrak{g}_{\mathbb{R}}$ is $r=|\Omega|$.

Clearly $\alpha_{i}^{\prime}=0$ iff $i \in I_{X}$, and for $j, k \in I \backslash I_{X}, \Delta^{\prime} \ni \alpha_{j}^{\prime}=\alpha_{k}^{\prime}$ iff $j$ and $k$ are in the same $\tau$-orbit. In particular, the set $\Pi^{\prime}:=\left\{\alpha_{i}^{\prime} ; i \in \Omega\right\}$ is a root basis of $\Delta^{\prime}$. We denote $\Delta_{+}^{\prime}$ (resp. $\Delta_{-}^{\prime}$ ) the set of positive (resp. negative) roots of $\Delta^{\prime}$ with respect to the basis $\Pi^{\prime}$. We have $\Delta^{\prime}=\Delta_{+}^{\prime} \cup \Delta_{-}^{\prime}$ (disjoint union) and $\sigma\left(\Delta_{+}^{\prime}\right)=\omega^{\prime}\left(\Delta_{+}^{\prime}\right)=\Delta_{-}^{\prime}$. For $\alpha^{\prime} \in \Delta^{\prime}$, the root space $\mathfrak{g}_{\mathbb{R}, \alpha^{\prime}}$ associated to $\alpha^{\prime}$, with 
respect to adjoint action of $\mathfrak{a}_{\mathbb{R}}$, is finite dimensional. Let $\mathfrak{g}_{\mathbb{R}, 0}$ be the centralizer of $\mathfrak{a}_{\mathbb{R}}$ in $\mathfrak{g}_{\mathbb{R}}$ and $\mathfrak{n}_{ \pm}^{\prime}=\underset{\alpha^{\prime} \in \Delta_{ \pm}^{\prime}}{\bigoplus} \mathfrak{g}_{\mathbb{R}, \alpha^{\prime}}$, then we have

$$
\mathfrak{g}_{\mathbb{R}}=\mathfrak{n}_{-}^{\prime} \oplus \mathfrak{g}_{\mathbb{R}, 0} \oplus \mathfrak{n}_{+}^{\prime}
$$

Note that $\mathfrak{g}_{\mathbb{R}, 0}=\mathfrak{a}_{\mathbb{R}} \oplus\left(\mathfrak{k} \cap \mathfrak{g}_{\mathbb{R}, 0}\right)$ and $\sigma\left(\mathfrak{n}_{-}^{\prime}\right)=\mathfrak{n}_{+}^{\prime}$. It follows that $\mathfrak{n}_{-}^{\prime} \subset \mathfrak{k} \oplus \mathfrak{n}_{+}^{\prime}$. Hence one can deduce the Iwasawa decomposition

$$
\mathfrak{g}_{\mathbb{R}}=\mathfrak{k} \oplus \mathfrak{a}_{\mathbb{R}} \oplus \mathfrak{n}_{ \pm}^{\prime}
$$

3.1. The relative Weyl group. The new aspect to note here is that, as in Borcherd's work [11], a relative simple root $\alpha_{i}^{\prime}$ of the basis $\Pi^{\prime}$ may be imaginary (in the sense that no reflection corresponds to this root) and that is the case iff the subset $X(i):=X \cup\left\{\alpha_{i}, \alpha_{\tau i}\right\}$ of $\Pi$ is not of finite type; then all positive integer multiples of $\alpha_{i}^{\prime}$ are still roots. In the case where $X(i)$ is of finite type, the relative root $\alpha_{i}^{\prime}$ is said a real relative simple root. If $\alpha_{i}^{\prime}$ is real, then the (commutative) product of the longest elements, of the Weyl groups corresponding to the connected components of $X(i)$ meeting the $\tau$-orbit of $i$, commutes to $\sigma^{\prime}$ and $\sigma$ and induces on $\mathfrak{a}_{\mathbb{R}}$ a reflection $R_{i}^{\prime}$ such that

$$
R_{i}^{\prime}(a)=a-\left\langle\alpha_{i}^{\prime}, a\right\rangle \alpha_{i}^{\prime \check{i}} \quad\left(a \in \mathfrak{a}_{\mathbb{R}}\right)
$$

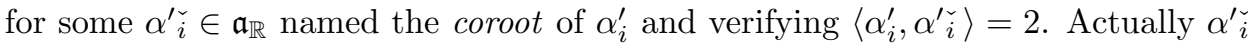
is the only element of $\mathfrak{a}$ (defined by the equations 2.2 .2 ) satisfying $\left\langle\alpha_{i}^{\prime}, \alpha_{i}^{\prime}\right\rangle=2$ and which is a linear combination of the $\alpha_{j}$, where $j$ runs over the union of the connected components of $X(i)$ meeting the $\tau$-orbit of $i$.

The relative Weyl group $W^{\prime}$ is generated by the reflections $R_{i}^{\prime}$ such that $\alpha_{i}^{\prime}$ is a real simple relative root. It is isomorphic to $N^{\prime} / H^{\prime}$, where $N^{\prime}$ (resp. $H^{\prime}$ ) is the normalizer (resp. the centralizer) of $\mathfrak{a}_{\mathbb{R}}$ in $G_{\mathbb{R}}$, and it is simply transitive on the set of minimal parabolic subalgebras of $\mathfrak{g}_{\mathbb{R}}$ of sign $\epsilon$ and containing $\mathfrak{a}_{\mathbb{R}}$.

If $\alpha_{i}^{\prime}$ is a real simple relative root, then $\Delta_{+}^{\prime} \cap \mathbb{Z}^{+} \alpha_{i}^{\prime} \subset\left\{\alpha_{i}^{\prime}, 2 \alpha_{i}^{\prime}\right\}$ and $2 \alpha_{i}^{\prime}$ is a root iff $\tau(i) \neq i$ are contained in the same connected component of $X(i)$, or $\tau(i)=i$ and $\Delta_{X(i)}$ has a root with coefficient 2 on $\alpha_{i}$.

Remark 3.1.1. If $X=\emptyset$ and $\tau \neq 1$, then $\mathfrak{g}_{\mathbb{R}}$ is quasi-split and $\mathfrak{t}_{\mathbb{R}}:=\mathfrak{h}_{\mathbb{R}}^{\tau}=$ $\sum_{i \in I} \mathbb{R}\left(p_{i}+p_{\tau i}\right)$ is a maximal split toral subalgebra. In order to avoid any confusion, one notes with $\bar{Y}$ (instead of $Y^{\prime}$ ) the objects relating to the quasi-split form similar to those introduced above, in the general case, for almost split real forms. In particular, the relative root system $\bar{\Delta}$ is $\Delta\left(\mathfrak{g}, \mathfrak{h}^{\tau}\right)$ and the corresponding relative Weyl group $\bar{W}$ is generated by $\bar{R}_{i}$ such that the $\tau$-orbit $\bar{i}$ of $i$ is of finite type. If $J \subset I$ is of finite type and $\tau$-stable, we denote $\bar{J}:=J /(\tau)$ and $\bar{W}_{\bar{J}}$ the subgroup of $\bar{W}$ generated by $\bar{R}_{j}, j \in \bar{J}$. For $i \in \bar{I}$, set $\bar{\alpha}_{i}:=\sum_{j \in i} \alpha_{j} \in \mathfrak{t}_{\mathbb{R}}$; then $\bar{\alpha}_{i}$ is real iff $\left\langle\bar{\alpha}_{i}, \bar{\alpha}_{i}\right\rangle=\left\langle\alpha_{i}, \bar{\alpha}_{i}\right\rangle$ is positive $(=1$ or 2$)$.

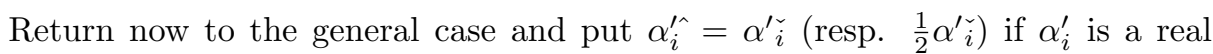
simple relative root such that $2 \alpha_{i}^{\prime} \notin \Delta^{\prime}\left(\right.$ resp. $\left.2 \alpha_{i}^{\prime} \in \Delta^{\prime}\right)$ and $\alpha_{i}^{\prime \prime}=\sum_{\bar{w} \in \bar{W}_{\bar{X}}} \bar{w}\left(\bar{\alpha}_{i}{ }^{\wedge}\right)$ if $\alpha_{i}^{\prime}$ is an imaginary simple relative root. Clearly $\alpha_{i}^{\prime \wedge} \in \mathfrak{a}_{\mathbb{R}}$ in the two cases. If $\alpha_{i}^{\prime}$ is a real simple relative root then $\alpha_{i}^{\prime}=\bar{p}_{i}-R_{i}^{\prime}\left(\bar{p}_{i}\right)$, where $\bar{p}_{i} \in \mathbb{R}\left(p_{i}+p_{\tau i}\right) \subset \mathfrak{a}_{\mathbb{R}}$ such that $\left\langle\alpha_{i}^{\prime}, \bar{p}_{i}\right\rangle=1$ and $R_{i}^{\prime}$ is the reflection corresponding to $\alpha_{i}^{\prime}$. Thus the expression of $\alpha_{i}^{\prime \wedge}$ results easily from that of $\alpha_{i}^{\prime \sim}$ in this case.

A relative simple root $\alpha_{i}^{\prime}$ is imaginary if and only if $\left\langle\alpha_{i}^{\prime}, \alpha_{i}^{\prime \wedge}\right\rangle \leq 0$. Here now some 
indications to calculate $\alpha_{i}^{\prime \wedge}$ when $\alpha_{i}^{\prime}$ is an imaginary simple relative root and the relative rank $r \geq 2$ (we don't calculate $\alpha_{i}^{\prime \wedge}$ when $r=1$ ). We will assume that $\alpha_{i}^{\prime}$ is imaginary of affine type (i.e. $X(i)$ is of affine type) and so $\left\langle\alpha_{i}^{\prime}, \alpha_{i}^{\prime \wedge}\right\rangle=0$. Note that this assumption is always checked for the hyperbolic Kac-Moody Lie algebras.

If the diagram automorphism $\tau$ (defining the $*$ - action of $\sigma^{\prime}$ ) is non trivial, then by considering the subset $\bar{X}(\bar{i})$ in $\bar{\Pi}$ (which is also of affine type) one can reduce the problem to the case $\tau=1$.

Assume now that $\tau=1$ and let $K_{X(i)}$ be the canonical central element of the affine Lie algebra $\mathfrak{g}_{X(i)}$, generated by $\left\{e_{\alpha}, f_{\alpha}, \alpha \in X(i)\right\}$ (see [17]; Chap. 6). Then one can write $K_{X(i)}=\sum_{\alpha \in X(i)} a_{\alpha} \alpha$, with positive integers $a_{\alpha}(\alpha \in X(i))$. In particular we have :

$$
\alpha_{i}^{\check{i}}=\frac{1}{a_{\alpha_{i}}}\left(K_{X(i)}-\sum_{\alpha \in X} a_{\alpha} \alpha^{\check{ }}\right)
$$

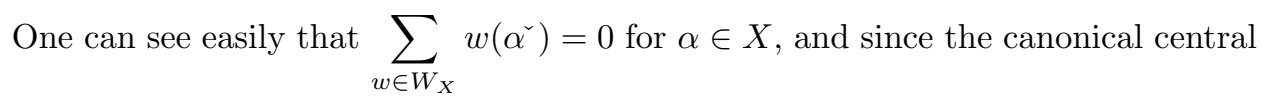
element $K_{X(i)}$ is fixed by $W_{X}$ (viewed as a subgroup of the affine Weyl group $W_{X(i)}$ ) we get :

$$
\alpha_{i}^{\prime \wedge}=\frac{\left|W_{X}\right|}{a_{\alpha_{i}}} K_{X(i)}
$$

3.2. The relative Kac-Moody matrix. In [[2], §3], Nicole Bardy associated to the relative root system $\Delta^{\prime}$ a relative Kac-Moody matrix $B^{\prime}:=\left(\left\langle\alpha_{j}^{\prime}, \alpha_{i}^{\prime \wedge}\right\rangle\right)=\left(b_{i, j}^{\prime}\right)$ which satisfies :

$$
\begin{aligned}
& b_{i, j}^{\prime} \in \mathbb{Z}, \\
& b_{i, i}^{\prime} \leq 2, \\
& b_{i, j}^{\prime} \in \mathbb{Z}^{-} \text {for } i \neq j, \\
& b_{i, j}^{\prime}=0 \text { iff } b_{j, i}^{\prime}=0 .
\end{aligned}
$$

Theorem 3.2.1 ([2; Théorème 3.10$])$ ). The relative root system is the only subset $\Delta^{\prime}$ of $\underset{i \in I^{\prime}}{\oplus} \mathbb{Z} \alpha_{i}^{\prime}$, where $I^{\prime}=\left(I \backslash I_{X}\right) /(\tau) \simeq \Omega$, such that :

(1) $\Delta^{\prime}=\Delta_{+}^{\prime} \cup \Delta_{-}^{\prime}$ where $-\Delta_{-}^{\prime}=\Delta_{+}^{\prime}=\Delta^{\prime} \cap\left(\underset{i \in I^{\prime}}{\oplus} \mathbb{Z}^{+} \alpha_{i}^{\prime}\right)$

(2) $\mathbb{Z}^{+} \alpha_{i}^{\prime} \cap \Delta_{+}^{\prime}= \begin{cases}\left\{\alpha_{i}^{\prime}\right\} & \text { if } \quad b_{i, i}^{\prime}=2 \\ \left\{\alpha_{i}^{\prime}, 2 \alpha_{i}^{\prime}\right\} & \text { if } b_{i, i}^{\prime}=1 \\ \mathbb{N}^{*} \alpha_{i}^{\prime} & \text { if } b_{i, i}^{\prime} \leq 0\end{cases}$

(3) For all $\alpha^{\prime} \in \Delta_{+}^{\prime} \backslash \Pi^{\prime}$, there exists $i \in I^{\prime}$ such that $\alpha^{\prime}-\alpha_{i}^{\prime} \in \Delta_{+}^{\prime}$.

(4) For all $i \in I^{\prime}$ and $\alpha^{\prime} \in \Delta_{+}^{\prime} \backslash \mathbb{N} \alpha_{i}^{\prime}$, the root subset $\Delta^{\prime} \cap\left(\alpha^{\prime}+\mathbb{Z} \alpha_{i}^{\prime}\right)$ is equal to :

(i) the string $\left\{\alpha^{\prime}-p \alpha_{i}^{\prime}, \ldots, \alpha^{\prime}+q \alpha_{i}^{\prime}\right\}$ with $p, q \in \mathbb{N}$ such that $p-q=\left(\frac{2}{b_{i, i}^{\prime}}\right)\left\langle\alpha^{\prime}, \alpha_{i}^{\prime \wedge}\right\rangle$ if $b_{i, i}^{\prime}>0$,

(ii) $\left\{\alpha^{\prime}\right\}$ if $b_{i, i}^{\prime} \leq 0$ and $\operatorname{supp}\left(\alpha^{\prime}\right)$ and $\left\{\alpha_{i}^{\prime}\right\}$ are not linked (with respect to $B^{\prime}$ ),

(iii) a set containing $\alpha^{\prime}+\mathbb{N} \alpha_{i}^{\prime}$ if $b_{i, i}^{\prime} \leq 0$ and $\operatorname{supp}\left(\alpha^{\prime}\right)$ and $\left\{\alpha_{i}^{\prime}\right\}$ are linked.

Remark 3.2.2. Actually when $\alpha_{i}^{\prime}$ is an imaginary relative simple root (i.e. $b_{i, i}^{\prime} \leq 0$ ) the relative root system $\Delta^{\prime}$ does'nt depend on the $\left\langle\alpha_{j}^{\prime}, \alpha_{i}^{\prime \wedge}\right\rangle=b_{i, j}^{\prime}\left(j \in I^{\prime}\right)$ but only on the fact they are zero or not. 
3.3. The relative Dynkin diagram. The normalized relative Kac-Moody matrix associated to $B^{\prime}$ is the matrix $A^{\prime}=\left(a_{i, j}^{\prime}\right)$ such that $a_{i, j}^{\prime}=\left(\frac{2}{b_{i, i}^{\prime}}\right) b_{i, j}^{\prime}=\left\langle\alpha_{j}^{\prime}, \alpha_{i}^{\prime}\right\rangle$ if $b_{i, i}^{\prime}>0$ and $a_{i, j}^{\prime}=b_{i, j}^{\prime}$ otherwise (see $[3] ; \S 2$ ).

To the relative Kac-Moody matrix is associated a graph $S\left(B^{\prime}\right)$, with $\left|I^{\prime}\right|$ vertices, called the relative Dynkin diagram as follows :

We associate to each $i$ a vertex equipped with a cross if $b_{i, i}^{\prime}=1$, with a sign - if $b_{i, i}^{\prime}<0$ and with 0 if $b_{i, i}^{\prime}=0$. Two vertices $i$ and $j$ are linked iff $b_{i, j}^{\prime}<0$; more precisely, if $b_{i, i}^{\prime}$ and $b_{j, j}^{\prime}$ are positive and $a_{i, j}^{\prime} a_{j, i}^{\prime}=n_{i, j} \leq 4$, the vertices $i$ and $j$ are joined by $m_{i, j}\left[:=\max \left(\left|a_{i, j}^{\prime}\right|,\left|a_{j, i}^{\prime}\right|\right)\right]$ line(s) with an arrow pointing towards the vertex $i$ if $\left|a_{i, j}^{\prime}\right|>1$. If $n_{i, j} \geq 5$ or one of the two coefficients $b_{i, i}^{\prime}$ or $b_{j, j}^{\prime}$ is non positive, the vertices $i$ and $j$ are joined by a thick line on which we write $\left|a_{i, j}^{\prime}\right|$ (beside the vertex $i$ ) and $\left|a_{j, i}^{\prime}\right|$ (beside the vertex $j$ ).

Concerning the nomenclature, the name $Z_{-}$stands for $\bullet_{-}$. The relative KacMoody matrix $\left(\begin{array}{cc}a & -s \\ -t & b\end{array}\right)$, with $a \geq b$ and $s \leq t$ if $a=b$, is named $H_{\left(s^{\prime}, t^{\prime}\right)}^{a, b}$, where $s^{\prime}=2 s\left(\right.$ resp. $\left.t^{\prime}=2 t\right)$ if $a=1$ (resp. $\left.b=1\right)$ and $s^{\prime}=s\left(\right.$ resp. $\left.t^{\prime}=t\right)$ otherwise. Actually, for the numbers $a, b$, the number 2 is omitted, 1 is replaced by ${ }^{\times}$and (for the Dynkin diagram) a strictly negative number is replaced by - . Some other names are given directly on the tables, they are inspired by the notations in table 2 and tables $3 \mathrm{a}, 3 \mathrm{~b}$ and $3 \mathrm{c}$ below.

\section{Classification}

Since the list of hyperbolic Kac-Moody Lie algebras seems to be very long (at least 136) we shall here restrict ourselves to the subclass of the strictly hyperbolic Kac-Moody Lie algebras and the (canonical) hyperbolic Lorentzian extensions of affine Lie algebras (see definition below) to classify their almost split real forms. The classification in the affine case was done in [2].

In the table 2 below we give, with their names, the list of all strictly hyperbolic symmetrizable Dynkin diagrams. The nomenclature is inspired from the book of Kac ([17], Exer. 4.3). It is noticed that the strictly hyperbolic Dynkin diagram $G^{\prime} G_{3}$ is missed in [31].

\section{Table 2}

List of strictly hyperbolic symmetrizable Dynkin diagrams

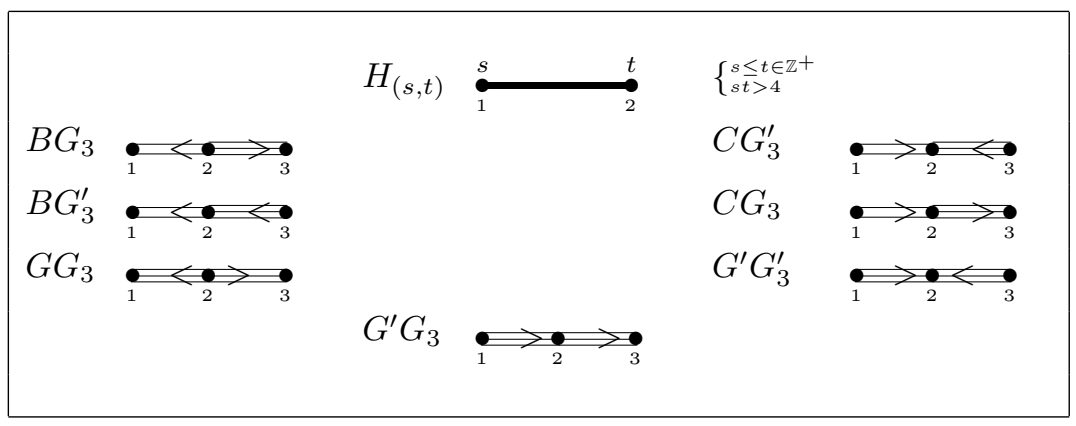


Table 2 (ended)

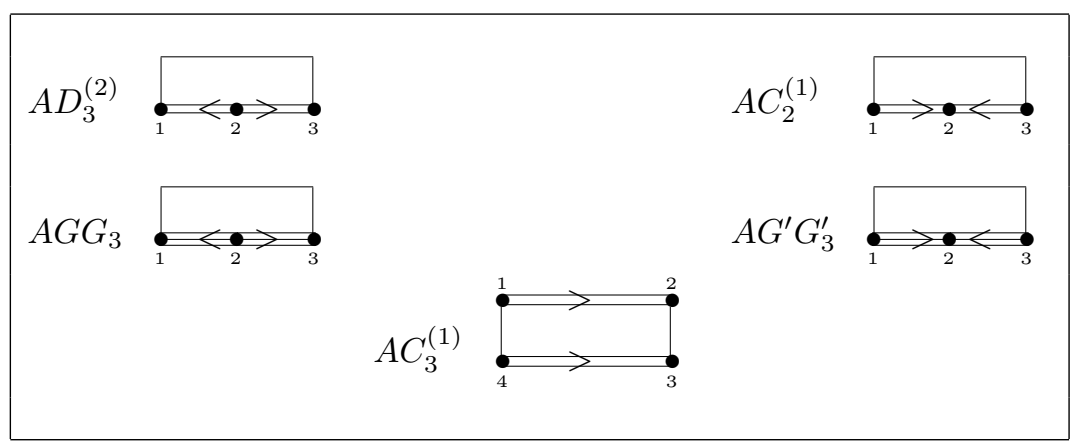

\subsection{Hyperbolic Lorentzian extensions of affine Lie algebras.}

Consider the Dynkin diagram of an affine Lie algebra of type $X_{n}^{(k)}$ with the vertices $\alpha_{0}, \alpha_{1}, \ldots, \alpha_{n_{k}}$. One extends the Dynkin diagram of $X_{n}^{(k)}$ by adding a root $\alpha_{-1}$ which is linked only to the root $\alpha_{0}$ (i.e. $\left\langle\alpha_{-1}, \alpha_{0}\right\rangle$ and $\left\langle\alpha_{0}, \alpha_{-1}^{-1}\right\rangle$ are negative integers, and $\left.\left\langle\alpha_{-1}, \alpha_{i}\right\rangle=0 \forall i=1,2, \ldots, n_{k}\right)$. Let $A$ be the generalized Cartan matrix corresponding to this extended Dynkin diagram, $\bar{A}$ the Cartan matrix corresponding to the finite type Dynkin diagram with the vertices $\alpha_{1}, \alpha_{2}, \ldots, \alpha_{n_{k}}$. We denote by $m$ the positive integer $\left\langle\alpha_{-1}, \alpha_{0}^{\check{0}}\right\rangle \times\left\langle\alpha_{0}, \alpha_{-1}\right\rangle$, then we have :

Proposition 4.1.1. The generalized Cartan matrix $A$ is symmetrizable, non singular and Lorentzian (i.e. the signature of the corresponding symmetrized matrix is $(++\ldots+-)$ ) and $\operatorname{det}(A)=-m \times \operatorname{det}(\bar{A})<0$.

Proof. The generalized Cartan matrix $A$ is clearly symmetrizable. Let $\mathfrak{g}$ be the corresponding symmetrizable Kac-Moody Lie algebra over the real field $\mathbb{R}, \mathfrak{h}:=$ $\mathbb{R} \alpha_{-1}^{\check{1}} \oplus \mathbb{R} \alpha_{0}^{\check{0}} \oplus \ldots \oplus \mathbb{R} \alpha_{n_{k}}^{\check{2}}$ the standard Cartan subalgebra and (. . .) the invariant bilinear form on $\mathfrak{g}$ (see 1.4). We have to show that the symmetric bilinear form $(.,$.$) has signature (++\ldots .+-)$ on $\mathfrak{h}$. Let $K$ be the canonical central element of the affine Lie algebra of type $X_{n}^{(k)}$ above (viewed as a subalgebra of $\mathfrak{g}$ ). Then one can see easily that

$$
\mathfrak{h}=\left(\mathbb{R} \alpha_{-1}^{\check{1}} \oplus \mathbb{R} K\right) \stackrel{\perp}{\oplus}\left(\mathbb{R} \alpha_{1} \oplus \ldots \oplus \oplus \mathbb{R} \alpha_{n_{k}}^{\check{r}}\right)
$$

where the orthogonal direct sum is taken relatively to the symmetric bilinear form $(.,$.$) on \mathfrak{h}$. As the Dynkin diagram corresponding to the vertices $\alpha_{1}, \alpha_{2}, \ldots, \alpha_{n_{k}}$ is of finite type, the symmetric bilinear form $\left(.\right.$, . .) is positive-definite on $\left(\mathbb{R} \alpha_{1} \oplus\right.$ $\left.\ldots \oplus \oplus \mathbb{R} \alpha_{n_{k}}\right)$. Since $(K, K)=0$ and $\left(\alpha_{-1}, K\right)=\left(\alpha_{-\check{1}}, \alpha_{0}\right)<0$, the matrix of the bilinear form $(.,$.$) on \left(\mathbb{R} \alpha_{-1}^{-} \oplus \mathbb{R} K\right)$, with respect to the basis $\left(\alpha_{-1}^{-}, K\right)$, takes the form $\left(\begin{array}{cc}a & -b \\ -b & 0\end{array}\right)$ where $a$ and $b$ are two positive real numbers. In particular the bilinear form $(.$, . $)$ has signature $(+-)$ on $\left(\mathbb{R} \alpha_{-1}^{\sim} \oplus \mathbb{R} K\right)$. Hence the symmetric bilinear form $(.,$.$) is nondegenerate on \mathfrak{h}$ and has signature $(++\ldots+-)$. The last formula for the determinant holds by describing the matrix $A$ using $\bar{A}$.

Definition 4.1.2. The Kac-Moody Lie algebra $\mathfrak{g}$ associated to the extended Dynkin Diagram above is called a Lorentzian extension of the affine Lie algebra $X_{n}^{(k)}$. 
The positive integer $m\left(=\left\langle\alpha_{-1}, \alpha_{0}^{\check{\gamma}}\right\rangle \times\left\langle\alpha_{0}, \alpha_{-1}^{-1}\right\rangle\right)$ is called the link multiplicity of the Lorentzian extension of $X_{n}^{(k)}$.

4.1.1. Remarks and notations.

1. Note that if the Kac-Moody algebra $\mathfrak{g}$ (Lorentzian extension of the affine Lie algebra $\left.X_{n}^{(k)}\right)$ is hyperbolic, then the link multiplicity $m \in\{1,2,3,4\}$ since the Dynkin diagram corresponding to the vertices $\alpha_{-1}$ and $\alpha_{0}$ (which will be noted $\left.D_{-1,0}\right)$ should be of finite or affine type.

2. The Lorentzian extensions of affine Lie algebras for which the two vertices $\alpha_{-1}$ and $\alpha_{0}$ are simply linked (i.e. $m=1$ ) are considered by M. Henneaux and B. Julia in [16] (see also [17]; §5.11) where they denoted by $X_{n}^{(k) \wedge}$ such a Lorentzian extension of an affine algebra of type $X_{n}^{(k)}$ and they called it the (standard or twisted) overextension of $X_{n}$ (depending on whether $k=1$ or $k=2,3$ ). Here we shall choose the notation $H X_{n}^{(k)}$ for the Lorentzian extension $X_{n}^{(k) \wedge}$ when this one is hyperbolic (and $m=1$ ) and it will be called the canonical hyperbolic Lorentzian extension of $X_{n}^{(k)}$

3. In the case $m=2$ or 3 (i.e. the rank-2 Dynkin diagram $D_{-1,0}$ is of type $C_{2}$ or $G_{2}$ ) we will denote by $H^{m} X_{n}^{(k)}$ (resp. $H^{m *} X_{n}^{(k)}$ ) the Lorentzian extension of $X_{n}^{(k)}$ if this one is hyperbolic and the root $\alpha_{-1}$ is shorter (resp. longer) than $\alpha_{0}$.

4. In the case $m=4$, the rank-2 Dynkin diagram $D_{-1,0}$ is of affine type and there are two possibilities:

i) The Dynkin diagram $D_{-1,0}$ is of type $A_{1}^{(1)}$ : the two roots $\alpha_{-1}$ and $\alpha_{0}$ have the same length and we denote by $H^{(1)} X_{n}^{(k)}$ the Lorentzian extension of $X_{n}^{(k)}$ if this one is hyperbolic.

ii) The Dynkin diagram $D_{-1,0}$ is of type $A_{2}^{(2)}$ : we denote by $H^{(2)} X_{n}^{(k)}$ (resp. $\left.H^{(2) *} X_{n}^{(k)}\right)$ the Lorentzian extension of $X_{n}^{(k)}$ if this one is hyperbolic and the root $\alpha_{-1}$ is shorter (resp. longer) than $\alpha_{0}$.

5. Note that for the twisted Dynkin diagram $A_{2 n}^{(2)}$ there exist two conventions for the root $\alpha_{0}$; so we consider the two corresponding canonical hyperbolic Lorentzian extensions $H A_{2 n}^{(2)}$ and $H^{\prime} A_{2 n}^{(2)}$, each one is the dual of the other. We adopt the same notations for the other hyperbolic Lorentzian extensions (with $m=2,3,4$ ) : $H^{x} A_{2 n}^{(2)}$ and $H^{\prime x} A_{2 n}^{(2)}, x=2,2 *, 3,3 *,(1),(2),(2) *$.

4.1.2. Canonical hyperbolic Lorentzian extensions of affine Lie algebras.

It was stated in [16] that all Lorentzian extensions (with $m=1$ ) of exceptional affine Lie algebras are hyperbolic (including $H A_{1}^{(1)}, H A_{2}^{(2)}, H^{\prime} A_{2}^{(2)}$ and $H D_{4}^{(3)}$ ); whereas there are some restrictions for those of classical affine Lie algebras.

In the Table 3a below we give, with their names, the list of all canonical hyperbolic Lorentzian extensions of classical affine Lie algebras other than $H A_{1}^{(1)}, H A_{2}^{(2)}$, $H^{\prime} A_{2}^{(2)}$ and $H D_{4}^{(3)}$ which we had regarded as exceptional . In particular, the four rank-10 hyperbolic Kac-Moody algebras which are often denoted $E_{10}, B E_{10}, C E_{10}$ and $D E_{10}$ are Lorentzian extensions of affine algebras and they are denoted here respectively $H E_{8}^{(1)}, H B_{8}^{(1)}, H A_{15}^{(2)}$ and $H D_{8}^{(1)}$. Note that table 3 a contains all the hyperbolic Dynkin diagrams of rank from 7 to 10 (see [17]; Exer. 4.3 or [31]). Finally, let us announce that the two rank- 5 hyperbolic Dynkin diagrams $H C_{3}^{(1)}$ and $H D_{4}^{(2)}$ are missed in [31]. 


\section{Table 3a}

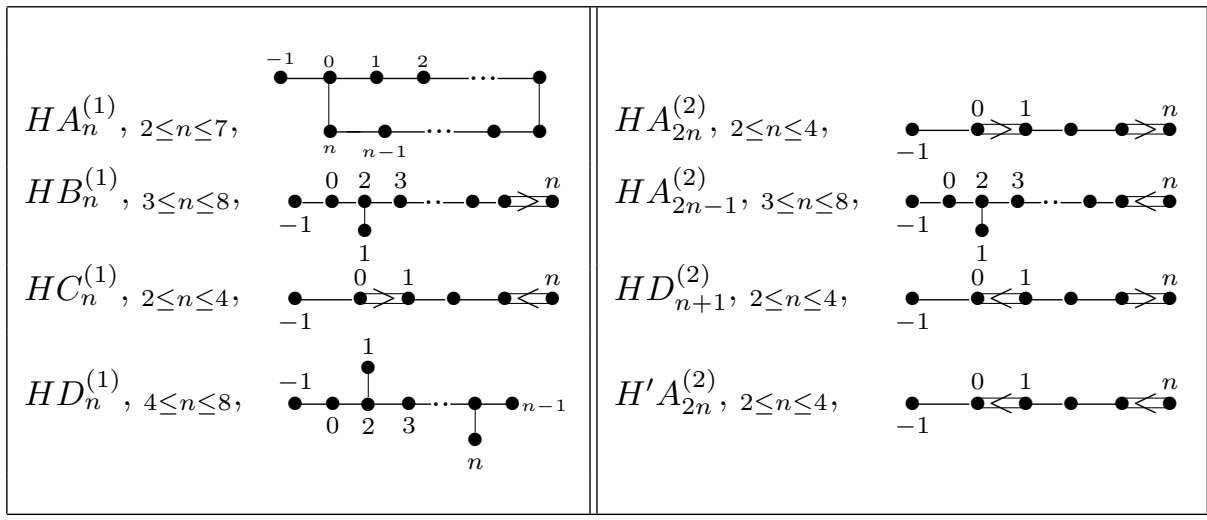

4.1.3. Hyperbolic Lorentzian extensions of affine Lie algebras with $m=2,3,4$.

The hyperbolic Lorentzian extensions of affine Lie algebras with $m=2,3$ are the following :

Table 3b

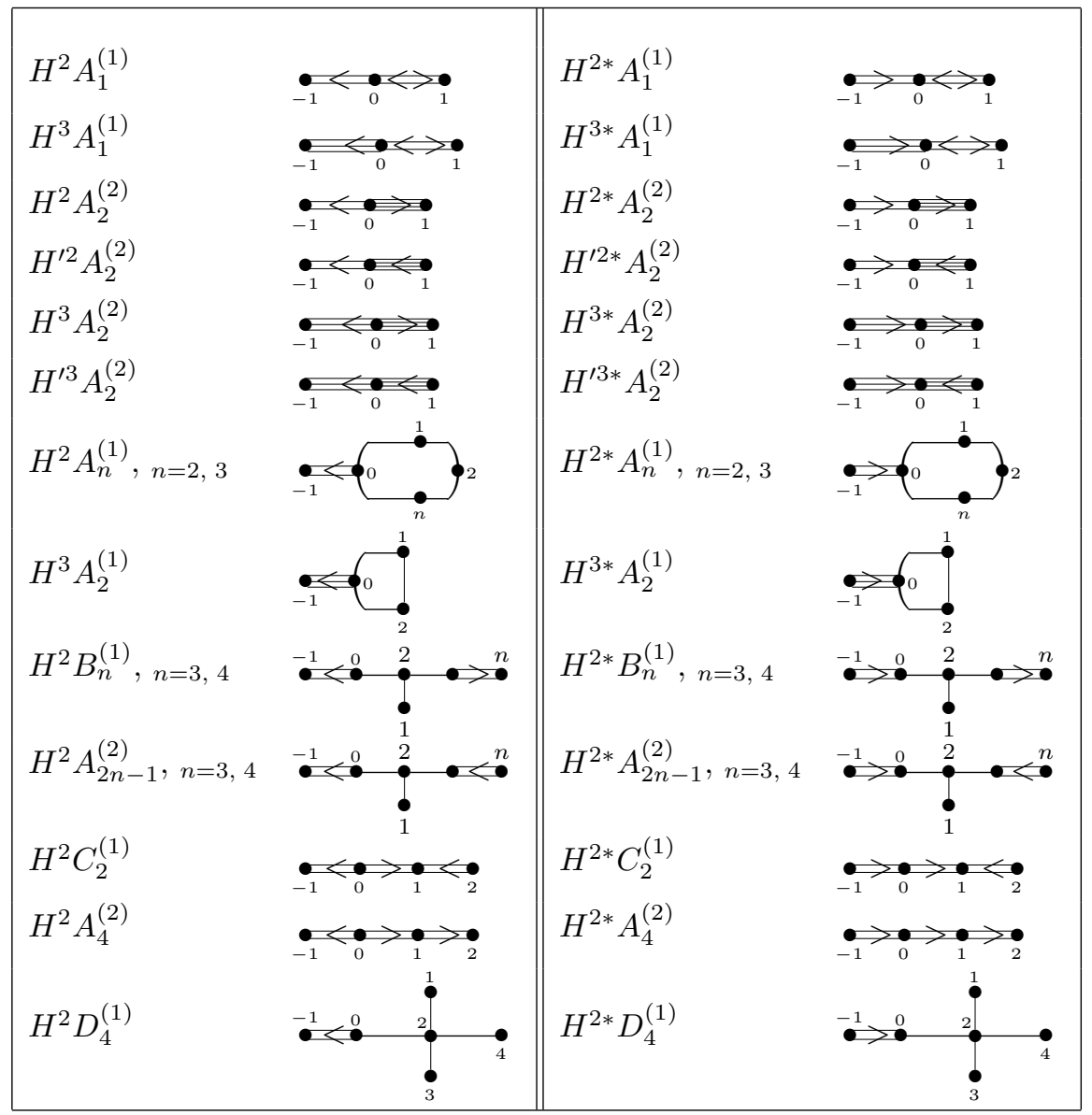


Table 3b (ended)

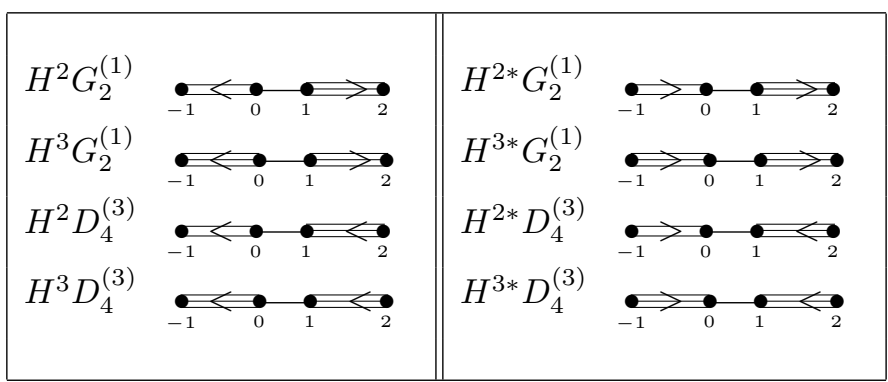

The other missed hyperbolic Lorentzian extensions are, if one changes the labeling, canonical or already enumerated with different names on the list above. In particular we have :

$$
\begin{aligned}
& H^{2} E_{8}^{(1)}=H B_{8}^{(1)} \\
& H^{2 *} E_{8}^{(1)}=H A_{15}^{(2)}
\end{aligned}
$$
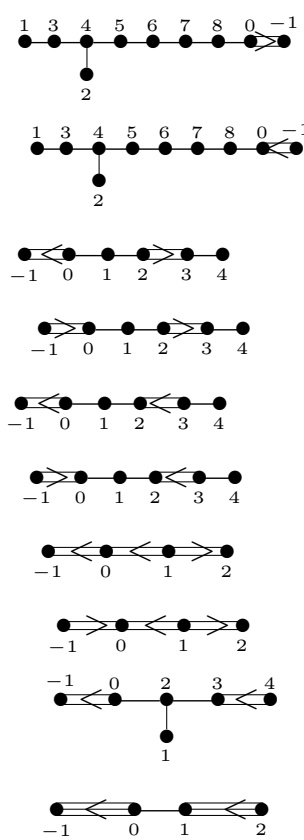

The hyperbolic Lorentzian extensions of affine Lie algebras, with $m=4$, are the following :

Table 3c

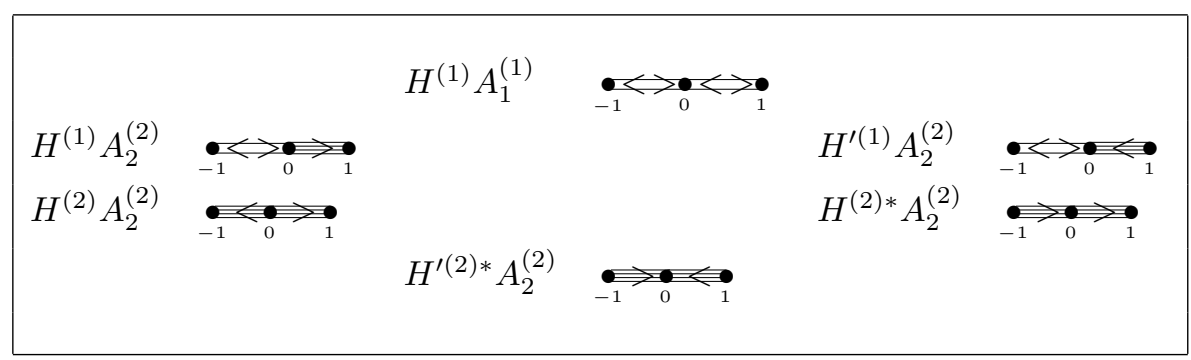


4.2. Tables. In the case of the strictly hyperbolic algebras and the canonical Lorentzian extensions of exceptional affine algebras (including $H A_{1}^{(1)}, H A_{2}^{(2)}, H^{\prime} A_{2}^{(2)}$ and $H D_{4}^{(3)}$ ) we indicate successively, in tables 4 and 5 , the name of the almost split real form, its Satake-Tits diagram (represented in accordance with 2.2.6), the conditions on the parameters and the relative root system (with its name and its Dynkin diagram). The name of the real form arises in this case in the form ${ }^{a} Z_{n, r}^{m}$ where $Z_{n}$ is the name of the hyperbolic algebra, $r=\left|I^{\prime}\right|$ is the relative rank, $a=1,2$ is the order of the diagram automorphism $\tau$ defining the $*$-action of $\sigma^{\prime}$ (omitted if it equals 1 ) and $\mathrm{m}$ (omitted if it equals 0 , i.e. in the quasi-split case) is the dimension of the Levi subalgebra $\mathfrak{l}^{\prime}=\mathfrak{g}_{X}$ (cf. 2.2.5).

In the case of the canonical hyperbolic Lorentzian extensions of classical affine algebras we indicate successively, in table 6 , the name of the almost split real form, its Satake-Tits diagram (represented in accordance with 2.2.6), the conditions on the parameters and the relative root system (with its name and its Dynkin diagram). The name of the real form arises in this case in the form ${ }_{i}^{a} Z_{n, r}$ where $Z_{n}$ is the name of the hyperbolic algebra, $a$ and $r$ are given according to same conventions as above, and $i$ (optional) is a parameter : it is often the cardinal of the smallest connected component of $X$.

The determination of all admissible indices is made using 2.6.3. Actually, when the relative rank is at least 2, this can also be deduced from Proposition 2.11 in [2] and the known lists of admissible indices for simple or affine Lie algebras (see [35] or [33] and [2]). The calculation of the relative Dynkin diagram is easy using the rules

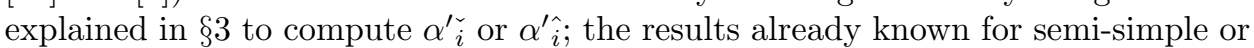
affine Lie algebras may also be used. We don't calculate $a_{i, i}^{\prime}$ in rank 1 (i.e. when $\left.b_{i, i}^{\prime}<0\right)$.

Finally, let us notice (as it will be shown in the tables below) that for the strictly hyperbolic almost split real forms, of rank $\geq 2$, the corresponding relative Dynkin diagrams are also strictly hyperbolic; whereas, for the hyperbolic canonical Lorentzian extensions of affine algebras, the relative Dynkin diagrams, associated to their almost split real forms of rank $\geq 3$, are hyperbolic but they are not always Lorentzian extensions of affine diagrams.

\section{Table 4}

List of almost split real forms of strictly hyperbolic symmetrizable Kac-Moody algebras

\begin{tabular}{|c|c|c|c|}
\hline Name & Satake-Tits index & Relative diagram & Name \\
\hline$H_{(s, t), 2}$ & $\stackrel{t}{t}$ & $\underset{2}{t}$ & $H_{(s, t)}$ \\
\hline$H_{(2 s, t), 1}^{3}$ & $\begin{array}{l}2 s \\
0 \\
1\end{array}$ & $\dot{\bullet}_{2}^{-}$ & $Z_{-}$ \\
\hline${ }^{2} H_{(s, s), 1}$ & $\underset{+}{S}$ & $\bullet_{1}^{\bullet}$ & $Z_{-}$ \\
\hline$B G_{3,3}$ & $0<0$ & $0<0$ & $B G_{3}$ \\
\hline$B G_{3,2}^{3}$ & $\underset{1}{0 \Longleftrightarrow{ }_{2}}$ & & $H_{(2,3)}$ \\
\hline$B G_{3,1}^{10}$ & 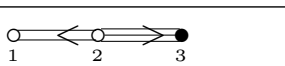 & $\bullet_{3}^{\bullet}-$ & $Z_{-}$ \\
\hline$B G_{3,1}^{14}$ & $0<\underset{1}{\stackrel{0}{2}>0}$ & $\bullet_{1}-$ & $Z_{-}$ \\
\hline
\end{tabular}


Table 4 (continued)

\begin{tabular}{|c|c|c|c|}
\hline Name & Satake-Tits index & Relative diagram & Name \\
\hline$C G_{3,3}^{\prime}$ & $\because><<$ & $\mapsto><\Leftrightarrow_{3}$ & $C G_{3}^{\prime}$ \\
\hline$C G_{3,1}^{\prime 14}$ & $\because \underset{2}{\longrightarrow}<0$ & $\bullet_{1}^{-}$ & $Z_{-}$ \\
\hline$C G_{3,1}^{\prime 6}$ & $\underset{1}{0} \gg<<0$ & $\dot{\bullet}_{2}-$ & $Z_{-}$ \\
\hline$B G_{3,3}^{\prime}$ & $0<0<0$ & $0<<<0$ & $B G_{3}^{\prime}$ \\
\hline$B G^{\prime 3,2}$ & $0<0<0$ & 1 & $H_{(1,6)}$ \\
\hline$B G_{3,1}^{\prime 10}$ & $0<0<3$ & $\bullet_{3}-$ & $Z_{-}$ \\
\hline$B G_{3,1}^{\prime 14}$ & $i<0<0$ & $\stackrel{\bullet}{i}-$ & $Z_{-}$ \\
\hline$C G_{3,3}$ & $\stackrel{i}{\longrightarrow} \gtrless_{3}$ & $\stackrel{\longrightarrow}{\longrightarrow} \gtrless_{3}$ & $C G_{3}$ \\
\hline$C G_{3,1}^{14}$ & $\Leftrightarrow \underset{2}{2} \Longrightarrow 0$ & $\stackrel{\bullet}{1}-$ & $Z_{-}$ \\
\hline$C G_{3,1}^{6}$ & $\stackrel{2}{\longrightarrow} \longrightarrow 0$ & $\dot{\bullet}_{2}-$ & $Z_{-}$ \\
\hline$G G_{3,3}$ & $\Leftrightarrow \Leftrightarrow$ & $\Leftrightarrow<{ }_{1}$ & $G G_{3}$ \\
\hline$G G_{3,1}^{14}$ & 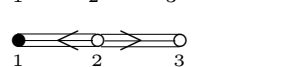 & $\dot{\bullet}_{1}^{-}$ & $Z_{-}$ \\
\hline$G G_{3,1}^{6}$ & $\Longleftrightarrow \Leftrightarrow$ & $\dot{ }_{2}^{-}$ & $Z_{-}$ \\
\hline${ }^{2} G G_{3,2}$ & $\stackrel{\square}{\Leftrightarrow} \Longleftrightarrow$ & $\frac{1}{2}$ & $H_{(1,6)}$ \\
\hline${ }^{2} G G_{3,1}^{3}$ & 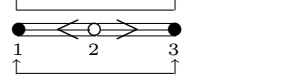 & $\bullet_{1}^{2}-$ & $Z_{-}$ \\
\hline$G^{\prime} G_{3,3}$ & $\Longleftrightarrow{ }_{2} \Rightarrow 3$ & $\Longleftrightarrow{ }_{2} \Longleftrightarrow$ & $G^{\prime} G_{3}$ \\
\hline$G^{\prime} G_{3,1}^{14}$ & $\Leftrightarrow \frac{0}{2}{ }_{3}$ & $\bullet_{1}-$ & $Z_{-}$ \\
\hline$G^{\prime} G_{3,1}^{6}$ & $\Longrightarrow 2$ & $\dot{ }_{2}-$ & $Z_{-}$ \\
\hline$G^{\prime} G_{3,1}^{14 *}$ & $\underset{2}{\Longleftrightarrow} \gtrless_{3}$ & $\stackrel{\bullet}{3}-$ & $Z_{-}$ \\
\hline$G^{\prime} G_{3,3}^{\prime}$ & $\Longleftrightarrow{ }_{1} \Longleftrightarrow$ & $\rightleftarrows{ }_{2}$ & $G^{\prime} G_{3}^{\prime}$ \\
\hline$G^{\prime} G_{3,1}^{\prime 14}$ & $\Longleftrightarrow{ }_{2} \Longleftrightarrow 0$ & $\bullet_{i}-$ & $Z_{-}$ \\
\hline$G^{\prime} G_{3,1}^{6}$ & $\Longrightarrow 2{ }_{3}$ & $\dot{\bullet}_{-}$ & $Z_{-}$ \\
\hline${ }^{2} G^{\prime} G^{\prime}{ }_{3,2}$ & $\Longrightarrow$ & 2 & $H_{(2,3)}$ \\
\hline${ }^{2} G^{\prime} G_{3,1}^{3}$ & $\Longrightarrow$ & $\bullet_{1}^{-}$ & $Z_{-}$ \\
\hline$A C_{2,3}^{(1)}$ & $\Longrightarrow 0$ & & $A C_{2}^{(1)}$ \\
\hline$A C_{2,2}^{(1) 3}$ & $\underset{1}{\longrightarrow}>_{2} \longleftrightarrow{ }_{3}$ & $\frac{4}{1}$ & $H_{(4,4)}$ \\
\hline
\end{tabular}


HECHMI BEN MESSAOUD

Table 4 (continued)

\begin{tabular}{|c|c|c|c|}
\hline Name & Satake-Tits index & Relative diagram & Name \\
\hline$A C_{2,1}^{(1) 10}$ & $\begin{array}{l}b \\
1\end{array}$ & $\bullet_{1}^{-}$ & $Z_{-}$ \\
\hline${ }^{2} A C_{2,2}^{(1)}$ & & $\mathrm{a}_{1}^{2} \quad \mathbf{H}_{1}^{4} \times$ & $H_{(2,4)}^{\times}$ \\
\hline${ }^{2} A C_{2,1}^{(1) 3}$ & $\sum_{2}=<$ & $\bullet_{1}^{-}$ & $Z_{-}$ \\
\hline${ }^{2} A C_{2,1}^{(1) 8}$ & $>p_{2}<$ & $\dot{\bullet}_{2}-$ & $Z_{-}$ \\
\hline$A D_{3,3}^{(2)}$ & & $\left\langle\bullet_{2}\right\rangle b_{3}$ & $A D_{3}^{(2)}$ \\
\hline${ }^{2} A D_{3,2}^{(2)}$ & & ${\underset{1}{1}}_{1}^{8} \times$ & $H_{(1,8)}^{\times}$ \\
\hline${ }^{2} A D_{3,1}^{(2) 3}$ & $<\underset{2}{0} \Rightarrow$ & $\bullet_{1}^{-}$ & $Z_{-}$ \\
\hline${ }^{2} A D_{3,1}^{(2) 8}$ & ${ }_{2} \quad 3$ & $\dot{\bullet}_{2}-$ & $Z_{-}$ \\
\hline$A G G_{3,3}$ & $<0$ & $\underset{1}{\Longleftrightarrow \Longleftrightarrow{ }_{3}}$ & $A G G_{3}$ \\
\hline$A G G_{3,1}^{14}$ & $\underset{2}{\langle 0}\rangle_{3}$ & & $Z_{-}$ \\
\hline${ }^{2} A G G_{3,2}$ & $\underset{2}{4}\rangle$ & ${\underset{1}{1}}_{1}^{12} \times$ & $H_{(1,12)}^{\times}$ \\
\hline${ }^{2} A G G_{3,1}^{3}$ & $\underset{2}{\leftarrow<}$ & $\dot{\bullet}_{-}$ & $Z_{-}$ \\
\hline${ }^{2} A G G_{3,1}^{8}$ & $\underset{1}{0} \underset{2}{2}$ & $\dot{\bullet}_{2}^{-}$ & $Z_{-}$ \\
\hline$A G^{\prime} G^{\prime}{ }_{3,3}$ & 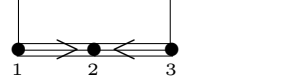 & $\underset{1}{\Longleftrightarrow}$ & $A G^{\prime} G^{\prime}{ }_{3}$ \\
\hline
\end{tabular}


Table 4 (ended)

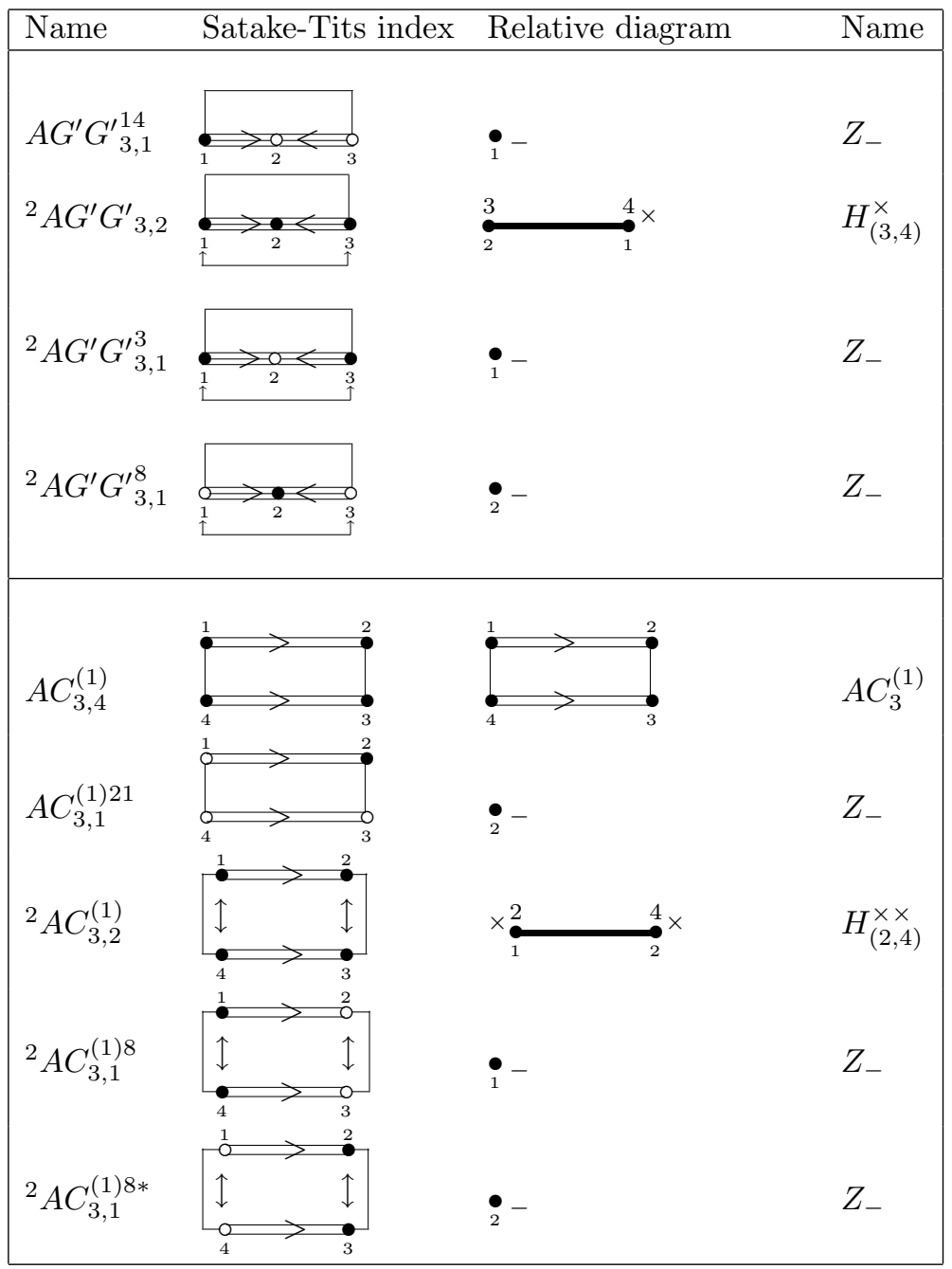

Table 5

List of almost split real forms for the canonical hyperbolic Lorentzian extensions of exceptional affine Kac-Moody algebras

\begin{tabular}{|c|c|c|c|}
\hline Name & Satake-Tits index & Relative diagram & Name \\
\hline$H A_{1,3}^{(1)}$ & $\stackrel{\bullet}{\bullet} \quad<>\bullet$ & 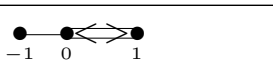 & $H A_{1}^{(1)}$ \\
\hline$H A_{1,2}^{(1) 3}$ & $\stackrel{\bullet}{-1} \quad<>0$ & ${ }_{-1}^{1} \quad \mathbf{0}_{0}^{2}$ & $H_{(1,2)}^{0}$ \\
\hline$H G_{2,4}^{(1)}$ & $2: \bullet$ & & $H G_{2}^{(1)}$ \\
\hline$H G_{2,2}^{(1) 14}$ & $\frac{0<0}{1} \quad \bullet \quad \bullet$ & $\mathbf{0}_{0}^{12} 0$ & $H_{(1,12)}^{0}$ \\
\hline$H F_{4,6}^{(1)}$ & & & $H F_{4}^{(1)}$ \\
\hline
\end{tabular}


HECHMI BEN MESSAOUD

Table 5 (continued)

\begin{tabular}{|c|c|c|c|}
\hline Name & Satake-Tits index & Relative diagram & Name \\
\hline$H F_{4,3}^{(1) 21}$ & 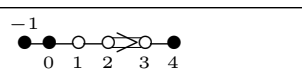 & $\underset{-1}{\Rightarrow} \bullet_{4}^{x}$ & $H^{2 *} A_{2}^{(2) \times}$ \\
\hline$H F_{4,2}^{(1) 36}$ & 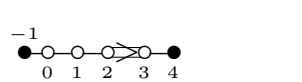 & $\overbrace{-1}^{1} \quad 2_{4}^{4}(4 !)$ & $H_{\left(1,2^{4} .4 !\right)}^{0}$ \\
\hline$H F_{4,2}^{(1) 52}$ & 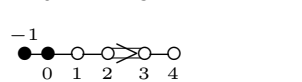 & & $H_{\left(1,2^{7} \cdot 3^{2}\right)}^{0}$ \\
\hline$H E_{6,8}^{(1)}$ & 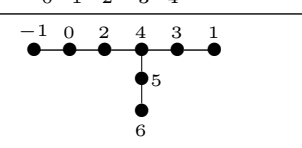 & 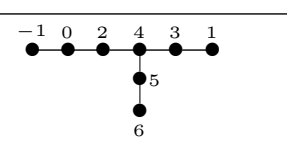 & $H E_{6}^{(1)}$ \\
\hline$H E_{6,4}^{(1) 28}$ & 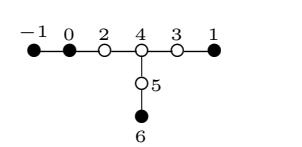 & $\rightarrow \sigma 0$ & $H^{2 *} A_{2}^{(1)}$ \\
\hline${ }^{2} H E_{6,6}^{(1)}$ & 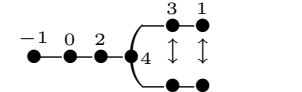 & $\bullet \bullet-1 \cdot \bullet$ & $H F_{4}^{(1)}$ \\
\hline${ }^{2} H E_{6,4}^{(1) 15}$ & 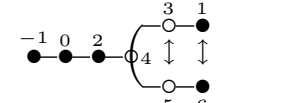 & $\stackrel{-1}{\bullet}:$ & $H A_{4}^{(2) \times}$ \\
\hline${ }^{2} H E_{6,2}^{(1) 45}$ & 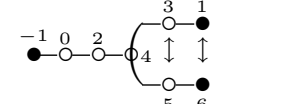 & $2^{4}(4 !)$ & $H_{\left(1,2^{4} .4 !\right)}^{0}$ \\
\hline${ }^{2} H E_{6,2}^{(1) 78}$ & 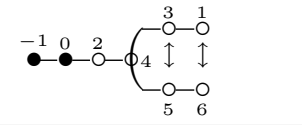 & $\mathbf{2}_{0}^{2^{7}} \cdot 3^{2}$ & $H_{\left(1,2^{7} \cdot 3^{2}\right)}^{0}$ \\
\hline$H E_{7,9}^{(1)}$ & 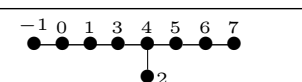 & 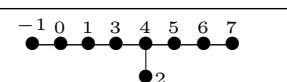 & $H E_{7}^{(1)}$ \\
\hline$H E_{7,6}^{(1) 9}$ & 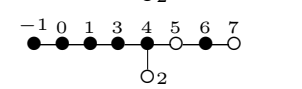 & & $H F_{4}^{(1)}$ \\
\hline$H E_{7,5}^{(1) 28}$ & 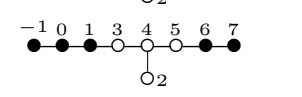 & $\stackrel{-1}{\bullet} \rightarrow$ & $H C_{3}^{(1)}$ \\
\hline$H E_{7,2}^{(1) 69}$ & 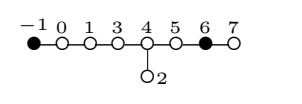 & $\underset{-1}{1}$ & $H_{\left(1,2^{5} .6 !\right)}^{0}$ \\
\hline$H E_{7,2}^{(1) 133}$ & 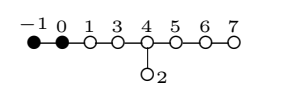 & $\begin{array}{l}2_{0}^{10} \cdot 3^{4} \cdot 5.7 \\
0\end{array}$ & $H_{\left(1,2^{10} .3^{4} .5 .7\right)}^{0}$ \\
\hline$H E_{7,1}^{(1) 248}$ & 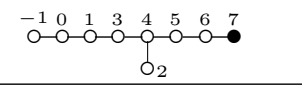 & $\bullet-$ & $Z_{-}$ \\
\hline$H E_{8,10}^{(1)}$ & $\bullet \cdot \bullet^{3} \bullet^{4} \cdot \bullet^{5} \cdot \bullet^{6} \cdot \bullet^{8} \cdot \bullet^{0} \bullet \bullet-1$ & $\bullet \cdot \bullet_{0}^{4} \cdot 0^{5} \cdot \bullet^{6} \cdot \bullet \bullet \bullet \bullet \bullet-1$ & $H E_{8}^{(1)}$ \\
\hline$H E_{8,6}^{(1) 28}$ & 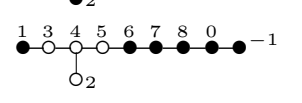 & $\because \because: \bullet>0$ & $H F_{4}^{(1)}$ \\
\hline$H E_{8,2}^{(1) 120}$ & 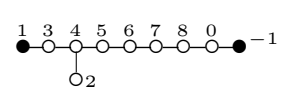 & 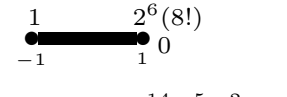 & $H_{\left(1,2^{6} .8 !\right)}^{0}$ \\
\hline$H E_{8,2}^{(1) 248}$ & 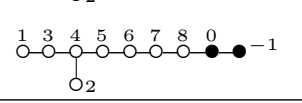 & 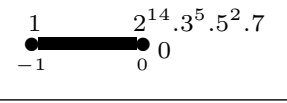 & $H_{\left(1,2^{14}, 3^{5} .5^{2} .7\right)}^{0}$ \\
\hline
\end{tabular}


Table 5 (ended)

\begin{tabular}{|c|c|c|c|}
\hline Name & Satake-Tits index & Relative diagram & Name \\
\hline$H A_{2,3}^{(2)}$ & $\stackrel{\bullet}{\bullet_{0}}$ & $\Longleftrightarrow$ & $H A_{2}^{(2)}$ \\
\hline$H A_{2,2}^{(2) 3}$ & $\stackrel{\bullet}{\Longrightarrow} \underset{0}{\rightleftharpoons}$ & $\underset{-1}{1}$ & $H_{(1,2)}^{0}$ \\
\hline$H^{\prime} A_{2,3}^{(2)}$ & $\underset{-1}{\bullet} \underset{0}{\bullet} \underset{1}{\bullet}$ & $\underset{-1}{\bullet} \underset{0}{\bullet} \underset{1}{\bullet}$ & $H^{\prime} A_{2}^{(2)}$ \\
\hline$H^{\prime} A_{2,1}^{(2) 6}$ & $\underset{-1}{\circ} \underset{0}{\infty}$ & $\bullet$ & $Z_{-}$ \\
\hline$H E_{6,6}^{(2)}$ & & & $H E_{6}^{(2)}$ \\
\hline$H E_{6,3}^{(2) 21}$ & & & $H A_{2}^{(2) \times}$ \\
\hline$H E_{6,2}^{(2) 52}$ & 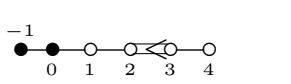 & $2^{7} \cdot 3^{2}$ & $H_{\left(1,2^{7} .3^{2}\right)}^{0}$ \\
\hline$H D_{4,4}^{(3)}$ & $\stackrel{\bullet}{\bullet} \quad \stackrel{0}{\overbrace{2}}$ & 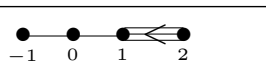 & $H D_{4}^{(3)}$ \\
\hline$H D_{4,2}^{(3) 14}$ & $\stackrel{\bullet-1}{\bullet} \underset{1}{\rightleftharpoons}$ & $\mathbf{0}_{0}^{12} 0$ & $H_{(1,12)}^{0}$ \\
\hline
\end{tabular}

Table 6

List of almost split real forms for the canonical hyperbolic Lorentzian extensions of classical affine Kac-Moody algebras

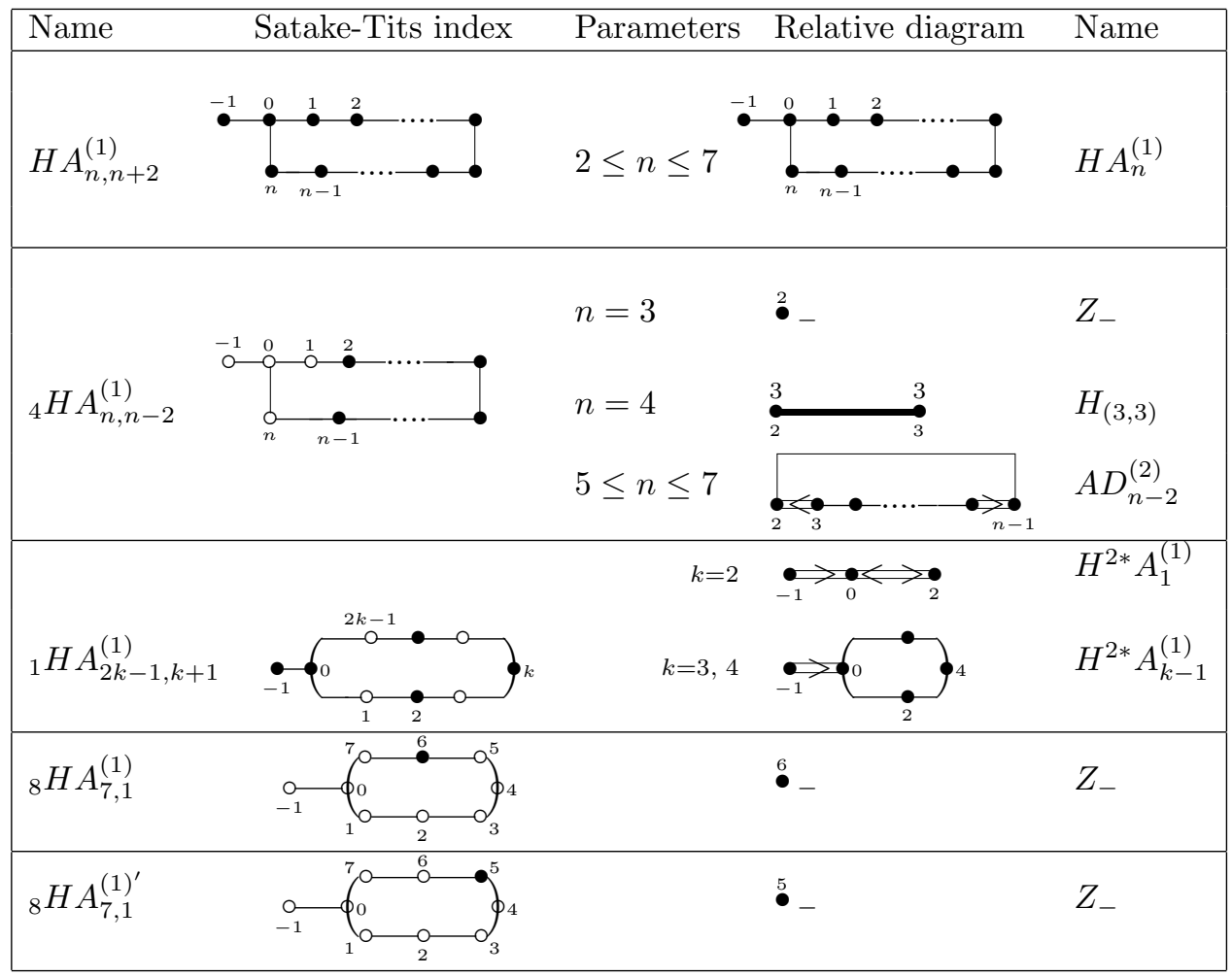


HECHMI BEN MESSAOUD

Table 6 (Continued)

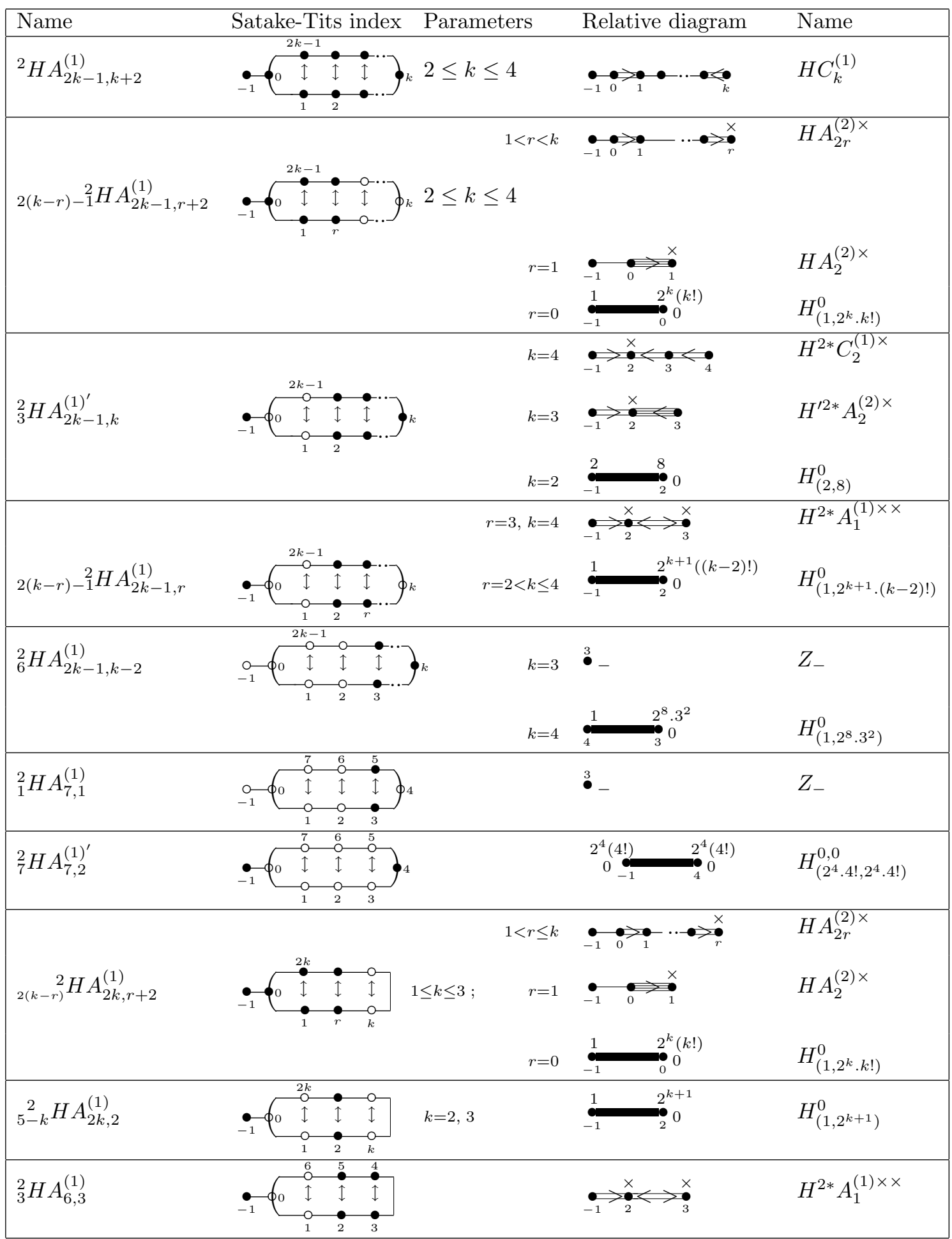


Table 6 (continued)

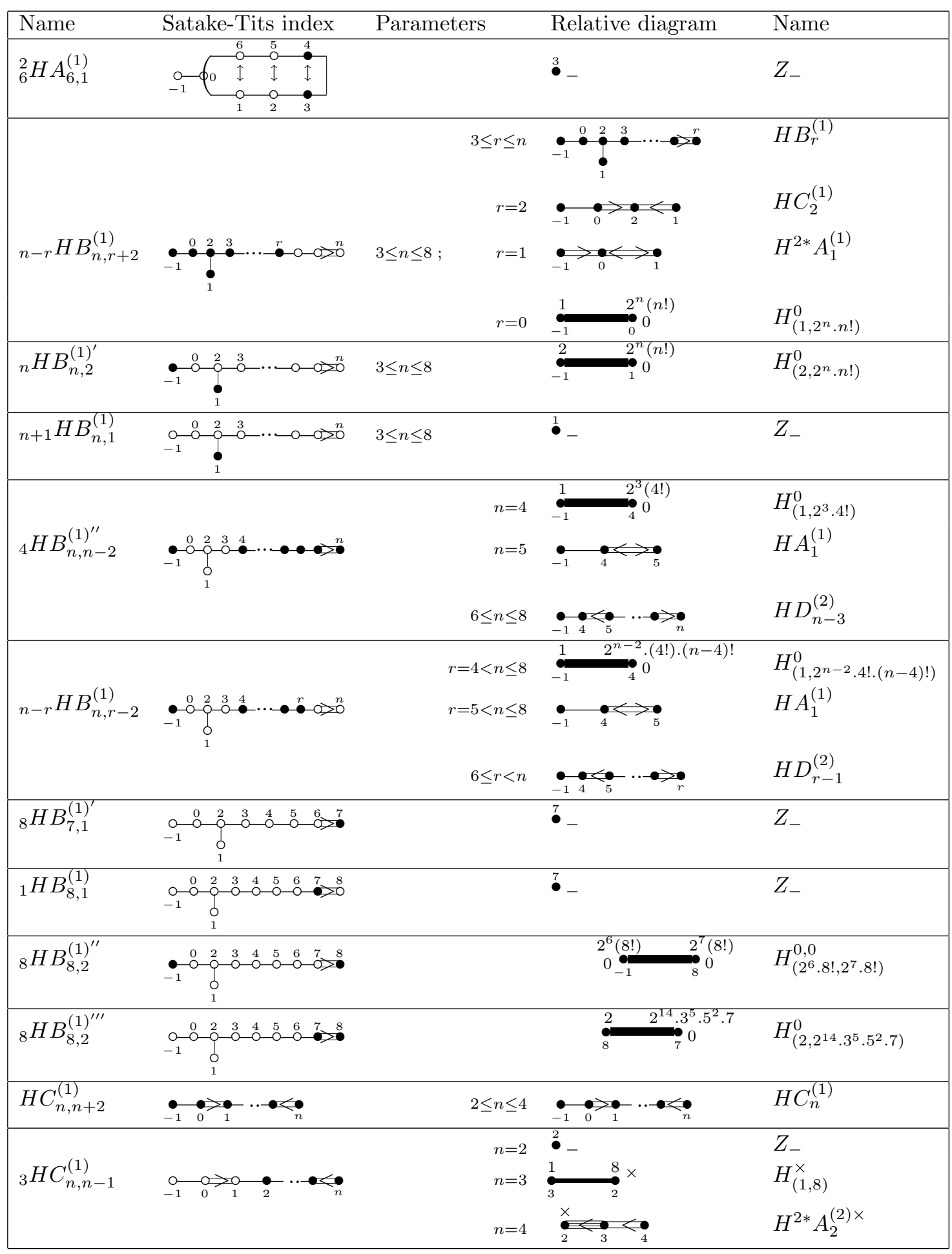


Table 6 (continued)

\begin{tabular}{|c|c|c|c|c|}
\hline Name & Satake-Tits index & Parameters & Relative diagram & Name \\
\hline${ }_{4} H C_{n, n-2}^{(1)}$ & 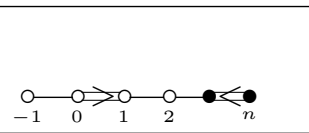 & $\begin{array}{l}n=3 \\
n=4\end{array}$ & $\begin{array}{l}\bullet_{-}^{-} \\
2^{8} \cdot 3^{2} \\
0\end{array}$ & $\begin{array}{l}Z_{-} \\
H_{\left(1,2^{8} \cdot 3^{2}\right)}^{0}\end{array}$ \\
\hline${ }_{1} H C_{n, 3}^{(1)}$ & $-1: 00 \quad: \quad 0<0$ & $\begin{array}{r}n=2 \\
n=3,4\end{array}$ & 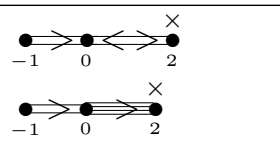 & $\begin{array}{l}H^{2 *} A_{1}^{(1) \times} \\
H^{2 *} A_{2}^{(2) \times}\end{array}$ \\
\hline $\begin{array}{l}{ }_{1} H C_{4,3}^{(1)^{\prime}} \\
{ }_{1} H C_{4,4}^{(1)} \\
{ }_{n} H C_{n, 2}^{(1)^{\prime}}\end{array}$ & 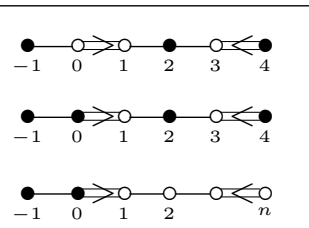 & $2 \leq n \leq 4$ & 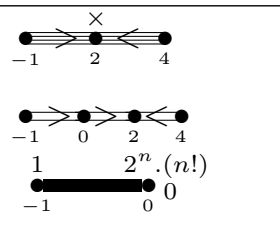 & $\begin{array}{l}H^{\prime(2) *} A_{2}^{(2) \times} \\
H^{2 *} C_{2}^{(1)} \\
H_{\left(1,2^{n} . n !\right)}^{0}\end{array}$ \\
\hline${ }_{n-2} H C_{n, 2}^{(1)}$ & $\therefore \quad 0<0<0<0$ & $\begin{array}{l}n=3 \\
n=4\end{array}$ & 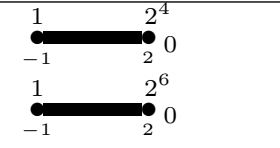 & $\begin{array}{l}H_{\left(1,2^{4}\right)}^{0} \\
H_{\left(1,2^{6}\right)}^{0}\end{array}$ \\
\hline${ }_{n} H C_{n, 2}^{(1)}$ & $\begin{array}{lll}-1 & 0>0 & 0\end{array}-0<0$ & $\begin{array}{l}n=2 \\
n=4\end{array}$ & 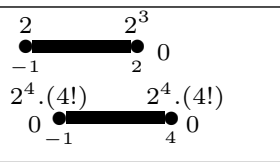 & $\begin{array}{l}H_{\left(2,2^{3}\right)}^{0} \\
H_{\left(2^{4} .4 !, 2^{4} .4 !\right)}^{0,0}\end{array}$ \\
\hline$H D_{n, n+2}^{(1)}$ & & $4 \leq n \leq 8$ & & $H D_{n}^{(1)}$ \\
\hline${ }_{n-r} H D_{n, r+2}^{(1)}$ & 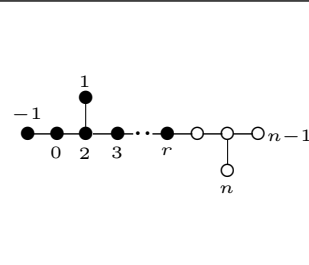 & $\begin{aligned} r=2, n \text { even } \\
4 \leq n \leq 8 \\
\quad \begin{aligned} 3 \leq r \leq n-2 \\
n-r \text { even }\end{aligned}\end{aligned}$ & 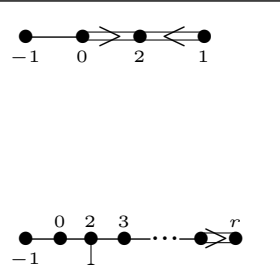 & $\begin{array}{l}H C_{2}^{(1)} \\
H B_{r}^{(1)}\end{array}$ \\
\hline${ }_{n-1} H D_{n, 3}^{(1)}$ & $\begin{array}{l}0 \\
1 \\
n\end{array}$ & $n=5,7$ & $\Leftrightarrow$ & $H^{2 *} A_{1}^{(1)}$ \\
\hline${ }_{n} H D_{n, 2}^{(1)}$ & 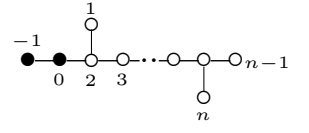 & $n=4,6,8$ & $\underset{-1}{1} \quad 2_{0}^{n-1} \cdot \mathbf{2}_{0}^{n-1} \cdot(n !)$ & $H_{\left(1,2^{n-1} . n !\right)}^{0}$ \\
\hline${ }_{n} H D_{n, 2}^{(1)^{\prime}}$ & 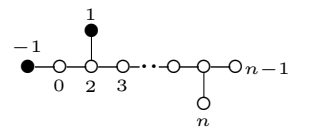 & $n=4,6,8$ & $\underset{-1}{2} \quad 2_{1}^{n-1} \cdot(n !)$ & $H_{\left(2,2^{n-1} \cdot n !\right)}^{0}$ \\
\hline${ }_{1} H D_{8,2}^{(1)}$ & • & & $\stackrel{1}{1}_{7} \quad 2_{6}^{11} \cdot 3^{4} \cdot 5.7$ & $H_{\left(1,2^{11} .3^{4} .5 .7\right)}^{0}$ \\
\hline${ }_{8} H D_{8,2}^{(1)^{\prime \prime}}$ & 0 & & $\begin{array}{ll}\stackrel{i}{i} & \stackrel{i}{i} \\
0 & \stackrel{8}{-1} \\
i=2^{7} .8 ! & \end{array}$ & $H_{(i, i)}^{0,0}$ \\
\hline
\end{tabular}


Table 6 (continued)

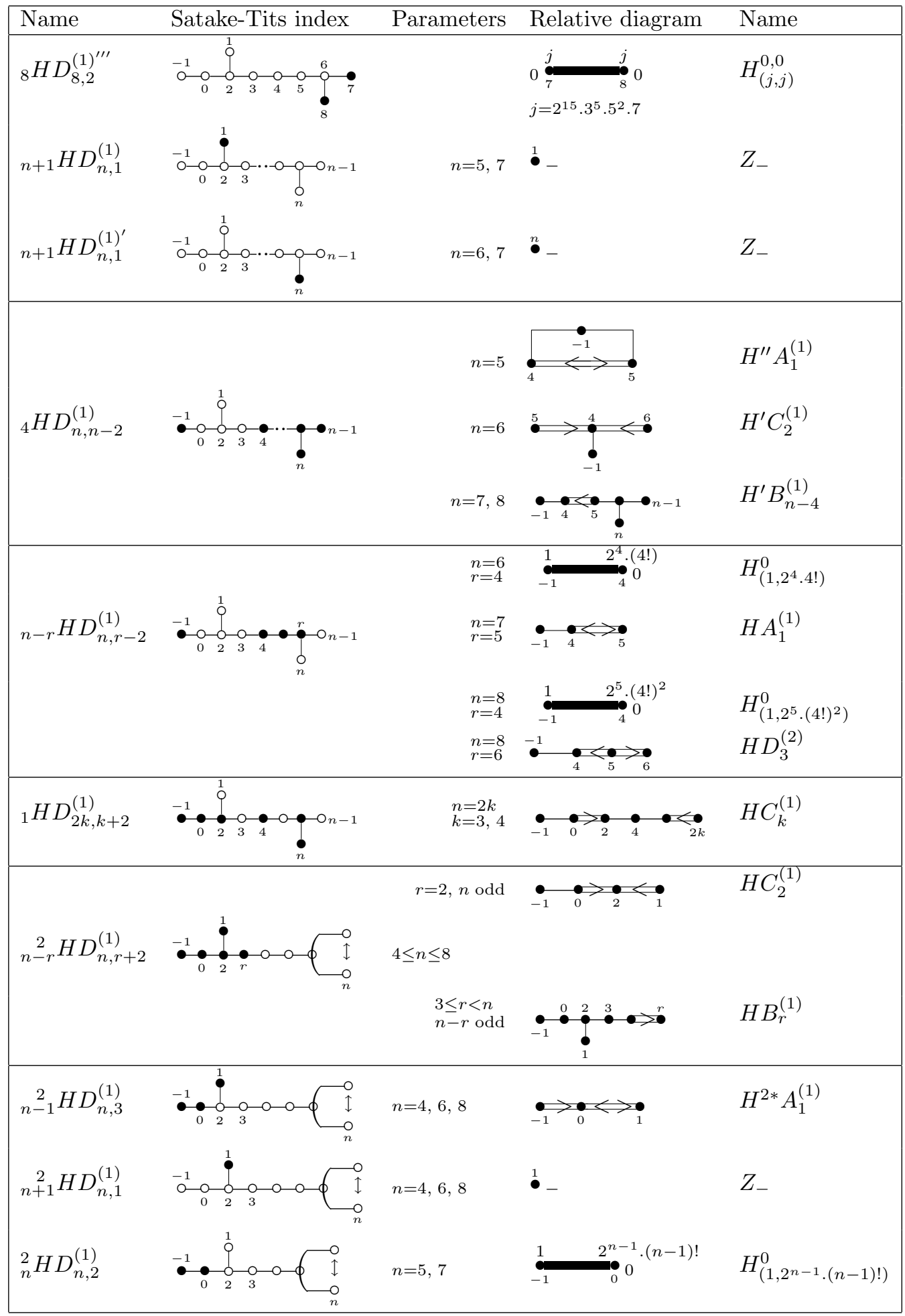


HECHMI BEN MESSAOUD

Table 6 (continued)

\begin{tabular}{|c|c|c|c|c|}
\hline Name & Satake-Tits index & Parameters & Relative diagram & Name \\
\hline${ }_{n}^{2} H D_{n, 2}^{(1)^{\prime}}$ & 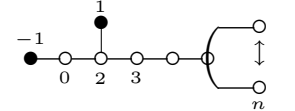 & $n=5,7$ & $\underset{-1}{2} \quad 2_{1}^{n-1} \cdot(n-1) !$ & $H_{\left(2,2^{n-1} \cdot(n-1) !\right)}^{0}$ \\
\hline${ }_{1}^{2} H D_{2 k+1, k+2}^{(1)}$ & 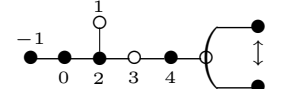 & $\begin{array}{l}n=2 k+1 \\
k=2,3\end{array}$ & 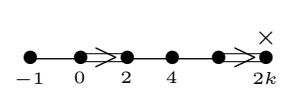 & $H A_{2 k}^{(2) \times}$ \\
\hline${ }_{4}^{2} H D_{n, n-3}^{(1)}$ & & $\begin{array}{r}n=5 \\
n=6 \\
n=7,8\end{array}$ & 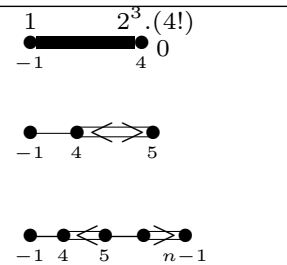 & $\begin{array}{l}H_{\left(1,2^{3} .4 !\right)}^{0} \\
H A_{1}^{(1)} \\
H D_{n-4}^{(2)}\end{array}$ \\
\hline${ }_{3}^{2} H D_{n, n-5}^{(1)}$ & & $\begin{array}{l}n=7 \\
n=8\end{array}$ & 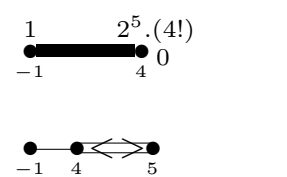 & $\begin{array}{l}H_{\left(1,2^{5} .4 !\right)}^{0} \\
H A_{1}^{(1)}\end{array}$ \\
\hline $\begin{array}{l}{ }_{7}^{2} H D_{7,1}^{(1)} \\
{ }_{8}^{2} H D_{8,1}^{(1)}\end{array}$ & 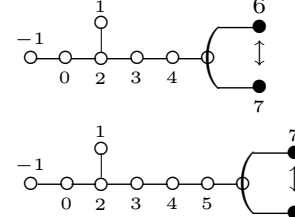 & & $\bullet_{-}$ & $\begin{array}{l}Z_{-} \\
Z_{-}\end{array}$ \\
\hline${ }_{k-r} H A_{2 k, r+2}^{(2)}$ & $\stackrel{\bullet}{\bullet} \rightarrow \mathbf{0}_{r}$ & $\begin{aligned} & r=0 \\
& 2 \leq k \leq 4 \\
& r=1 \\
& 2 \leq r \leq k\end{aligned}$ & 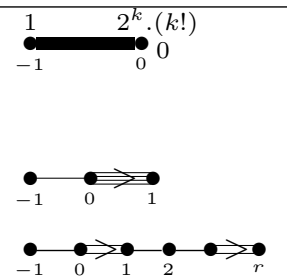 & $\begin{array}{l}H_{\left(1,2^{k} . k !\right)}^{0} \\
H A_{2}^{(2)} \\
H A_{2 r}^{(2)}\end{array}$ \\
\hline${ }_{3} H A_{2 k, k-1}^{(2)^{\prime}}$ & $\begin{array}{cccc}0 & 0>0 & \bullet & \bullet \\
-1 & 0 & 1 & 2\end{array}: \bullet$ & $\begin{array}{l}k=2 \\
k=3 \\
k=4\end{array}$ & 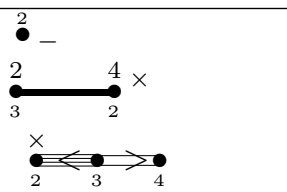 & $\begin{array}{l}Z_{-} \\
H_{(2,4)}^{\times} \\
H^{2} A_{2}^{(2) \times}\end{array}$ \\
\hline${ }_{1} H A_{8,2}^{(2)}$ & 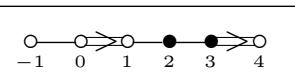 & & $\underbrace{2}_{3} \quad 4 x$ & $H_{(2,4)}^{\times}$ \\
\hline${ }_{k-2} H A_{2 k, 1}^{(2)}$ & 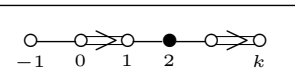 & $k=3,4$ & $\boldsymbol{e}_{-}$ & $Z_{-}$ \\
\hline${ }_{4} H A_{2 k, k-2}^{(2)^{\prime}}$ & 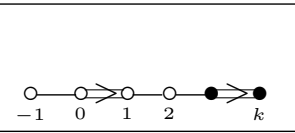 & $\begin{array}{l}k=3 \\
k=4\end{array}$ & $\begin{array}{ll}\mathbf{e}_{-} & \\
2 & 2^{7} \cdot 3^{2} \\
\mathbf{a}^{2} & 3_{0}\end{array}$ & $\begin{array}{l}Z_{-} \\
H_{\left(2,2^{7} \cdot 3^{2}\right)}^{0}\end{array}$ \\
\hline${ }_{1} H A_{8,1}^{(2)}$ & $\underset{-1}{0} 0_{0}^{0}>0$ & & $3_{-}$ & $Z_{-}$ \\
\hline$H^{\prime} A_{2 k, k+2}^{(2)}$ & 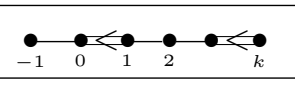 & $2 \leq k \leq 4$ & 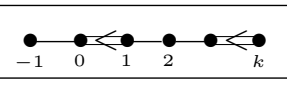 & $H^{\prime} A_{2 k}^{(2)}$ \\
\hline
\end{tabular}


Table 6 (continued)

\begin{tabular}{|c|c|c|c|c|}
\hline Name & Satake-Tits index & Parameters & Relative diagram & Name \\
\hline${ }_{4} H^{\prime} A_{2 k, k-2}^{(2)}$ & $\begin{array}{cccccc}0 & 0<0 & 0 & 0< & 0 & 0 \\
-1 & 0 & 1 & 2 & \end{array}$ & $\begin{array}{l}k=3 \\
k=4\end{array}$ & $\frac{1}{1}$ & $\begin{array}{l}Z_{-} \\
H_{\left(1,2^{8} .3^{2}\right)}^{0}\end{array}$ \\
\hline${ }_{k} H^{\prime} A_{2 k, 2}^{(2)^{\prime}}$ & 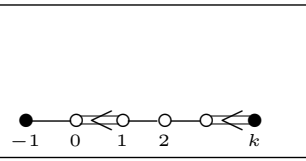 & $\begin{array}{l}k=3 \\
k=4\end{array}$ & 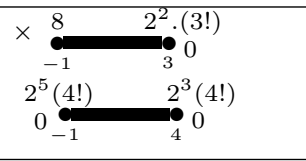 & $\begin{array}{l}H_{\left(8,2^{2} .3 !\right)}^{\times, 0} \\
H_{\left(2^{3} .4 !, 2^{5} .4 !\right)}^{0,0}\end{array}$ \\
\hline${ }_{1} H^{\prime} A_{2 k, 2}^{(2)}$ & 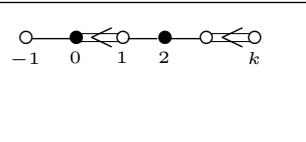 & $\begin{array}{r}k=2 \\
k=3,4\end{array}$ & $\underset{0}{\overbrace{0}^{8} \times}{ }_{0}^{4} \times$ & $\begin{array}{l}H_{(1,8)}^{\times} \\
H_{(2,4)}^{\times \times}\end{array}$ \\
\hline${ }_{1} H^{\prime} A_{2 k, 1}^{(2)}$ & 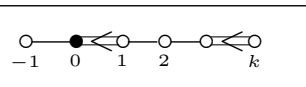 & $2 \leq k \leq 4$ & $\bullet_{-}$ & $Z_{-}$ \\
\hline$k-2 H^{\prime} A_{2 k, 1}^{(2)^{\prime}}$ & 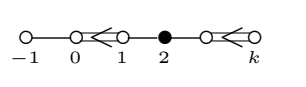 & $k=3,4$ & $?_{-}^{2}$ & $Z_{-}$ \\
\hline${ }_{3} H^{\prime} A_{4,1}^{(2)}$ & $0-0<0<0$ & & $\stackrel{2}{\bullet}_{-}$ & $Z_{-}$ \\
\hline${ }_{1} H^{\prime} A_{8,3}^{(2)}$ & 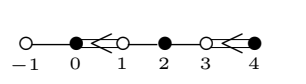 & & $\stackrel{\times}{=} \underset{2}{=0}$ & $H^{2 *} A_{2}^{(2) \times}$ \\
\hline${ }_{3} H^{\prime} A_{8,3}^{(2)}$ & $\begin{array}{llcccc}\bullet & 0 & 0 & 0 & 0 & 0\end{array}$ & & 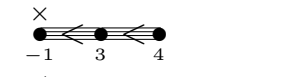 & $H^{(2) *} A_{2}^{(2) \times}$ \\
\hline${ }_{1} H^{\prime} A_{8,2}^{(2)^{\prime}}$ & $\begin{array}{cccccc}0 & 0<0 & 0 & 0< \\
-1 & 0 & 1 & 2 & 3 & 0\end{array}$ & & $\underset{0}{2_{2}^{4}}$ & $H_{\left(1,2^{4} .3 !\right)}^{0}$ \\
\hline$H A_{2 k-1, k+2}^{(2)}$ & 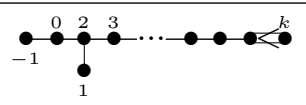 & $3 \leq k \leq 8$ & 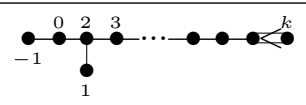 & $H A_{2 k-1}^{(2)}$ \\
\hline${ }_{1} H A_{2 k-1, r+2}^{(2)}$ & 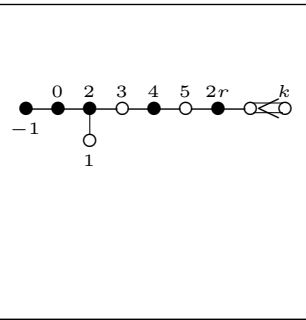 & $\begin{array}{r}r=1 \\
3 \leq k \leq 8 \\
2<2 r<k \\
2 r=k\end{array}$ & 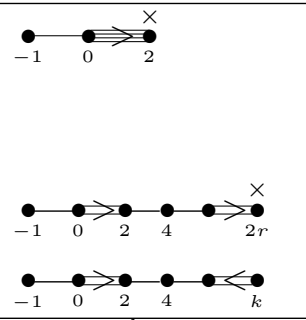 & $\begin{array}{l}H A_{2 r}^{(2) \times} \\
H C_{r}^{(1)}\end{array}$ \\
\hline${ }_{k} H A_{2 k-1,2}^{(2)}$ & 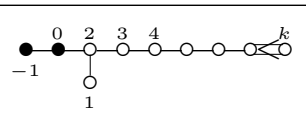 & $3 \leq k \leq 8$ & $\begin{array}{cc}1 & 2^{k} \cdot(k !) \\
-1 & 0\end{array}$ & $H_{\left(1,2^{k} . k !\right)}^{0}$ \\
\hline${ }_{4} H A_{2 k-1, k-2}^{(2)}$ & 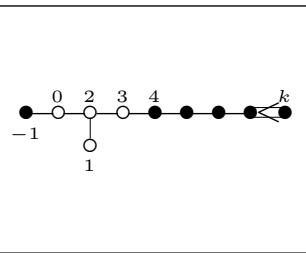 & $\begin{array}{l}k=4 \\
k=5\end{array}$ & $\underset{-1}{2}$ & $\begin{array}{l}H_{\left(2,2^{2} .4 !\right)}^{0} \\
H^{\prime} A_{2}^{(2)} \\
H^{\prime} A_{2(k-4)}^{(2)}\end{array}$ \\
\hline$k-6 A_{2 k-1,1}^{(2)}$ & 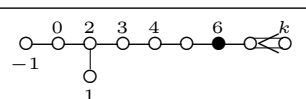 & $k=7,8$ & $\bullet_{-}$ & $Z_{-}$ \\
\hline${ }_{k+1} H A_{2 k-1,1}^{(2)}$ & 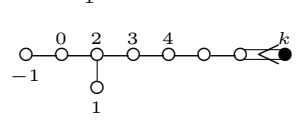 & $k=6,7$ & $\stackrel{k}{\bullet}$ & $Z_{-}$ \\
\hline
\end{tabular}


Table 6 (ended)

\begin{tabular}{|c|c|c|c|c|}
\hline Name & Satake-Tits index & Parameters & Relative diagram & Name \\
\hline${ }_{8} H A_{15,2}^{(2)^{\prime}}$ & 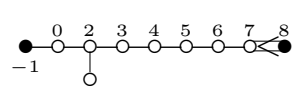 & & 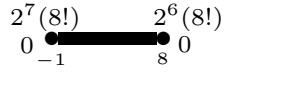 & $H_{\left(2^{6} .8 !, 2^{7} .8 !\right)}^{0,0}$ \\
\hline${ }_{1} H A_{15,2}^{(2)}$ & 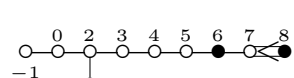 & & $\frac{2^{11} \cdot 3^{4} \cdot 5 \cdot 7}{0.7}$ & $H_{\left(1,2^{11} 3^{4} .5 .7\right)}^{0}$ \\
\hline${ }_{8} H A_{15,2}^{(2)}$ & 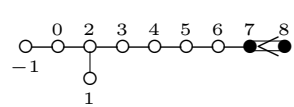 & & $2^{15} \cdot 3^{5} \cdot 5^{2} .7$ & $H_{\left(1,2^{15} .3^{5} \cdot 5^{2} .7\right)}^{0}$ \\
\hline & & $r=0$ & $n \cdot(n !)$ & $H_{\left(1,2^{n} . n !\right)}^{0}$ \\
\hline & & $r=1$ & -1 & $H A_{1}^{(1)}$ \\
\hline & & $2 \leq r \leq n$ & 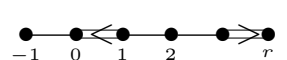 & $H D_{r+1}^{(2)}$ \\
\hline & & $n=3$ & $\underbrace{23}_{3} \cdot{ }_{0}^{(3 !)}$ & $H_{\left(4,2^{3} .3 !\right)}^{\times, 0}$ \\
\hline${ }_{3} H D_{n+1, n-1}^{(2)^{\prime}}$ & 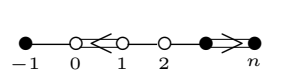 & $n=4$ & $\stackrel{x}{0}<<$ & $H^{(1)} A_{2}^{(2) \times}$ \\
\hline${ }_{4} H D_{n+1, n-2}^{(2)^{\prime}}$ & 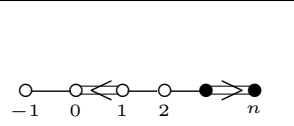 & $\begin{array}{l}n=3 \\
n=4\end{array}$ & $\begin{array}{l}0^{3}- \\
2^{7} \cdot 3^{2}\end{array}$ & $\begin{array}{l}Z_{-} \\
H_{\left(2,2^{7} \cdot 3^{2}\right)}^{0}\end{array}$ \\
\hline${ }_{1} H D_{5,2}^{(2)}$ & 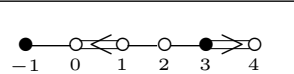 & & $2_{3}^{3} \cdot(3 !)$ & $H_{\left(4,2^{3} .3 !\right)}^{0}$ \\
\hline${ }_{4} H D_{5,2}^{(2)^{\prime \prime}}$ & 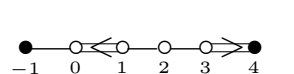 & & $\begin{array}{c}2^{4}(4 !) \\
0-1\end{array}$ & $H_{\left(2^{4} .4 !, 2^{4} .4 !\right)}^{0,0}$ \\
\hline${ }_{1} H D_{5,1}^{(2)}$ & 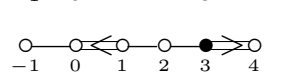 & & $3_{-}$ & $Z_{-}$ \\
\hline
\end{tabular}

Acknowledgments. I wish to thank Guy Rousseau for extremely useful suggestions and discussions that we had on this subject. I also thank him for his collaboration and his hospitality during my scientific stays at the Institute Elie Cartan of Nancy.

\section{REFERENCES}

[1] N. Andruskiewitsch; Some forms of Kac-Moody algebras. J. of Algebra 147 (1992) 324-344.

[2] V. Back-Valente, N. Bardy-Panse, H. Ben Messaoud et G. Rousseau; Formes presque déployées d'algèbres de Kac-Moody, Classification et racines relatives. J. of Algebra 171 (1995) 43-96.

[3] N. Bardy-Panse; Systèmes de racine infinis. Mémoire de la S.M.F 65 (1996).

[4] P. Batra; Invariants of real forms of affine Kac-Moody Lie algebras. J. of Algebra 223 (2000) 208-236.

[5] J. Bausch; Etude et classification des automorphismes d'ordre fini et de première espèce des algèbres de Kac-Moody affines. Revue de l'Institut Elie Cartan 11, Nancy (1988) 5-124.

[6] J. Bausch et G. Rousseau; Involutions de première espèce des algèbres de Kac-Moody affines, Revue de l'Institut Elie Cartan 11, Nancy (1988) 125-139.

[7] H. Ben Messaoud et G. Rousseau; Classification des formes réelles presque compactes des algèbres de Kac-Moody affines, J. of Algebra 267 (2003) 443-513. [Coquilles corrigées dans J. of Algebra 279 (2004) 409-412.] 
[8] H. Ben Messaoud et G. Rousseau; Sous-algèbres de Cartan des algèbres de Kac-Moody affines réelles presque compactes. Preprint Nancy (2004).

[9] H. Ben Messaoud et G. Rousseau; Sous-algèbres de Cartan des algèbres de Kac-Moody réelles presque déployées. À paraître au Journal of the Mathematical Society of Japon.

[10] S. Berman and A. Pianzola; Generators and relations for real forms of some Kac-Moody Lie algebras. Comm. in Algebra 15 (1987) 935-959.

[11] R. E. Borcherds; Generalized Kac-Moody algebras. J. of Algebra 115 (1988) 501-512.

[12] N. Bourbaki; Groupes et algèbres de Lie, Chap 4, 5 et 6 , Paris.

[13] T. Damour, M. Henneaux, B. Julia and H. Nicolai; Hyperbolic Kac-Moody algebras and chaos in Kaluza-Klein models. Phys. Lett. B 509 (2001) p. 323.

[14] P. Goddard and D.I. Olive; Algebras, lattices and strings, in : Vertex Operators in Mathematics and Physics, MSRI Publication, Vol 3, Springer-Verlag, Berlin, 1984, p.51.

[15] S. Helgason; Differential geometry, Lie groups and symmetric spaces. Academic Press, New York, 1978

[16] M. Henneaux and B. Julia; Hyperbolic billiards of pure $D=4$ supergravities. J. High Energy Physics JHEP 05 (2003) 47.

[17] V.G. Kac; Infinite dimensional Lie algebras. Third edition, Cambridge University Press (1990).

[18] V.G. Kac and D.H. Peterson; On geometric invariant Theory of infinite dimensional groups. In "Algebraïc groups" Utrecht 1986, Springer lecture note in Math. 1271 (1987) 109-142.

[19] V.G. Kac and S.P. Wang; On automorphisms of Kac-Moody algebras and groups. Advances in Math. 92 (1992) 129-195.

[20] J. Lepowsky and R.V. Moody; Hyperbolic Lie algebras and quasi-regular cusps on Hilbert modular surfaces. Math. ann. 245 (1979) p. 63.

[21] F. Levstein; A classification of involutive automorphisms of an affine Kac-Moody Lie algebra. J. of Algebra 114 (1988) 489-518.

[22] R.G. Matthias, D.I. Olive and P.C. West; A class of Lorentzian Kac-Moody algebras. Nucl. Phys. B 645 (2002) 403-437.

[23] R.V. Moody; Root systems of hyperbolic type. Advances in Mathematics 33, 144-160 (1979).

[24] K. C. Pati, D. Parashar, and S. Kaushal; Involutive automorphisms and Iwasawa decomposition of some hyperbolic Kac-Moody algebras. J. Math. Phys. Volume 40. Number 1 (1998) 501-510.

[25] D.H. Peterson and V.G. Kac; Infinite flag varieties and conjugacy theorems. Proc. Natl. Acad. Sc. USA 80 (1983) 1778-1782.

[26] G. Rousseau; Formes réelles presque compactes des algèbres de Kac-Moody affines. Revue de l'Institut Elie Cartan 11, Nancy (1988) 175-205.

[27] G. Rousseau; Formes réelles presque déployées des algèbres de Kac-Moody affines. In "Harmonic Analysis" Luxembourg 1987, Springer lecture note in Math. 1359 (1988) 252-264.

[28] G. Rousseau; Almost split $K$-forms of Kac-Moody Algebras. In "Infinite dimensional Lie algebras and groups" Marseille (1988), V.G. Kac éditeur, Adv. Ser. in Math. Physics 7, World Scientific (1989) 70-85.

[29] G. Rousseau; L'immeuble jumelé d'une forme presque déployée d'une algèbre de Kac-Moody. Bull. Soc. Math. Belg. 42 (1990) 673-694.

[30] G. Rousseau; On forms of Kac-Moody algebras. Proceedings of Symposia in pure Mathematics, 56 part 2 (1994) 393-399.

[31] C. Saçlioğlu; Dynkin diagrams for hyperbolic Kac-Moody algebras. J. Phys. A 22, 3753(1989).

[32] I. Satake; On the theory of reductive algebraic groups over a perfect field. J. Math. Soc. Japan 15(1963) 210-235.

[33] I. Satake; Classification theory of semi-simple algebraic groups. Lecture note in pure and applied math. 3, Marcel Dekker, New-York, 1971.

[34] I. Schnakenburg and P. west; Kac-Moody symmetries of IIB supergravity. Phys. Lett. B 517 (2001) p. 421.

[35] J. Tits; Classification of algebraic semi-simple groups. In "Algebraic groups and discountinuous subgroups" Boulder 1965, Proc. of Symposia in pure Math. IX (1966) 33-62.

[36] J. Tits; Normalisateurs de Tores, groupes de Coxeter étendus. J. of Algebra 4 (1966) 96-116.

Departement de Mathématiques, Faculté des Sciences, 5000 Monastir. Tunisie.

E-mail address: hechmi.benmessaoud@fsm.rnu.tn 